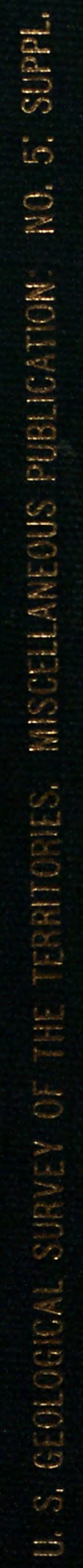



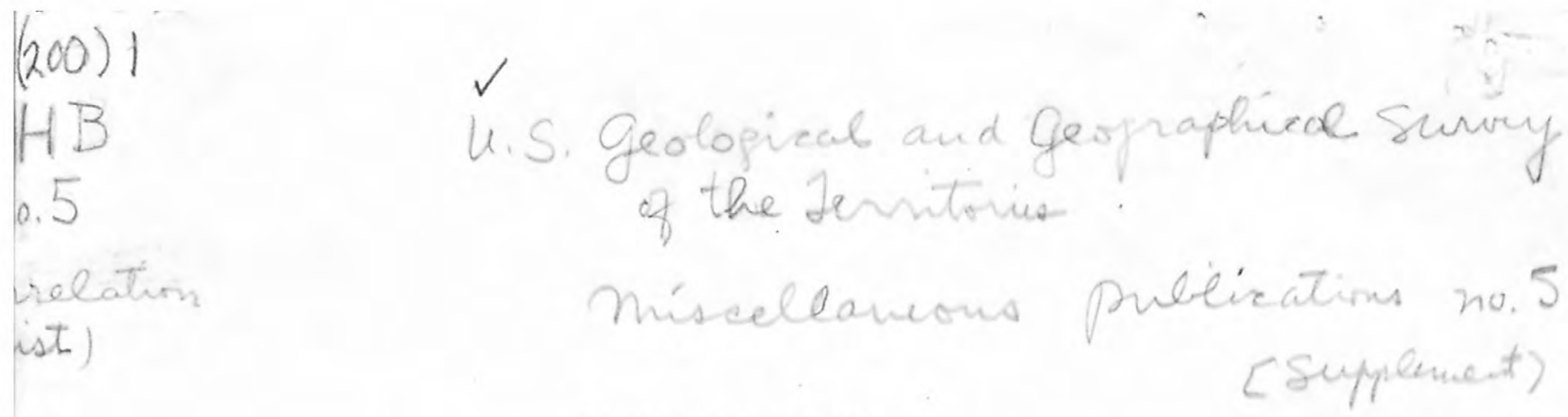

Correlation III

of

Now and 01d Nhmbers

of

The Desertptive Catalogra of the Photographs of the U. So Geologlcal Strrvey of the torrttorle for the roar 1869 to 1873. ML scellanow ous Publd cation Ho, 5. Department of the Intorlor, $U_{0} S_{0}$ Geologloul Survey of the Tersico tor10s。 1876

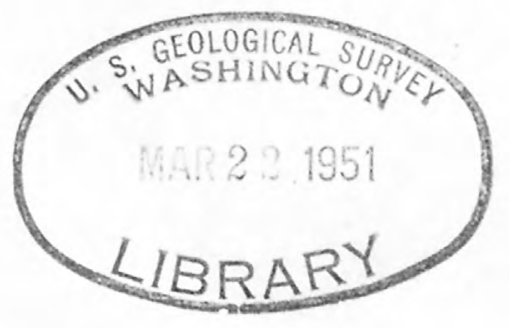

Fhotographs o Il brary

U. S. Goologtonl Surver March 2951

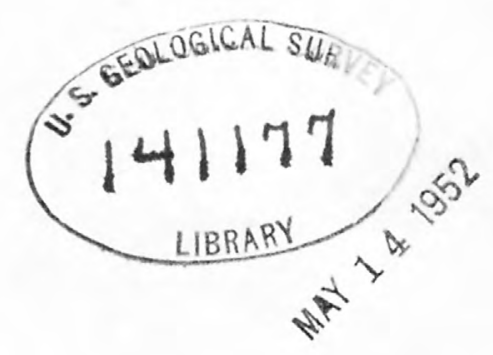




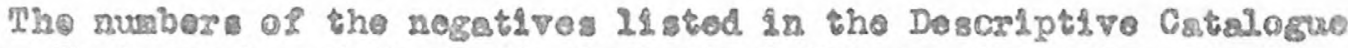
of the Fhotographs of the Uo So Goologleal Shrrvey of the Torrd torles

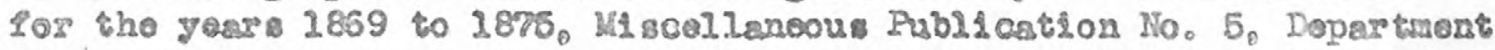

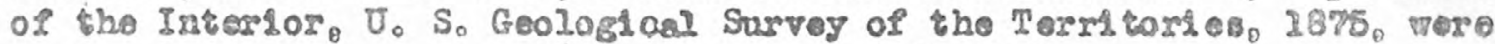
soplaced nany yoars ago with new mbers. The following is st of new numbers corrolating the 0.1 mumbers has boen prepared to sorve as a key to the 1875 âtalog.

Tach asignod now mabor reprogent a negativo or a print in our collectlon and the sequenco of now mbers lollows the osder of priats mounted In Plve albuas in the photographtc 11 brary of the U. So Goologtanl survey. Blank maces in the 118 indicate unknom old catalog mubers and. dâฺ.

The glass negative axe the 5 origine colbums are stoud

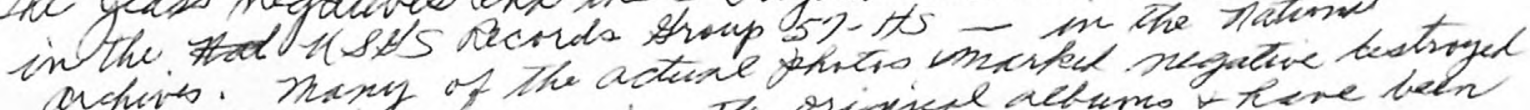

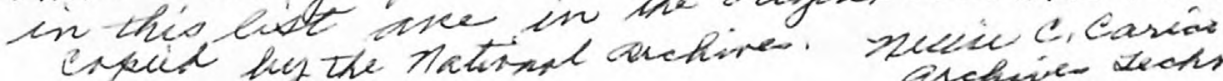

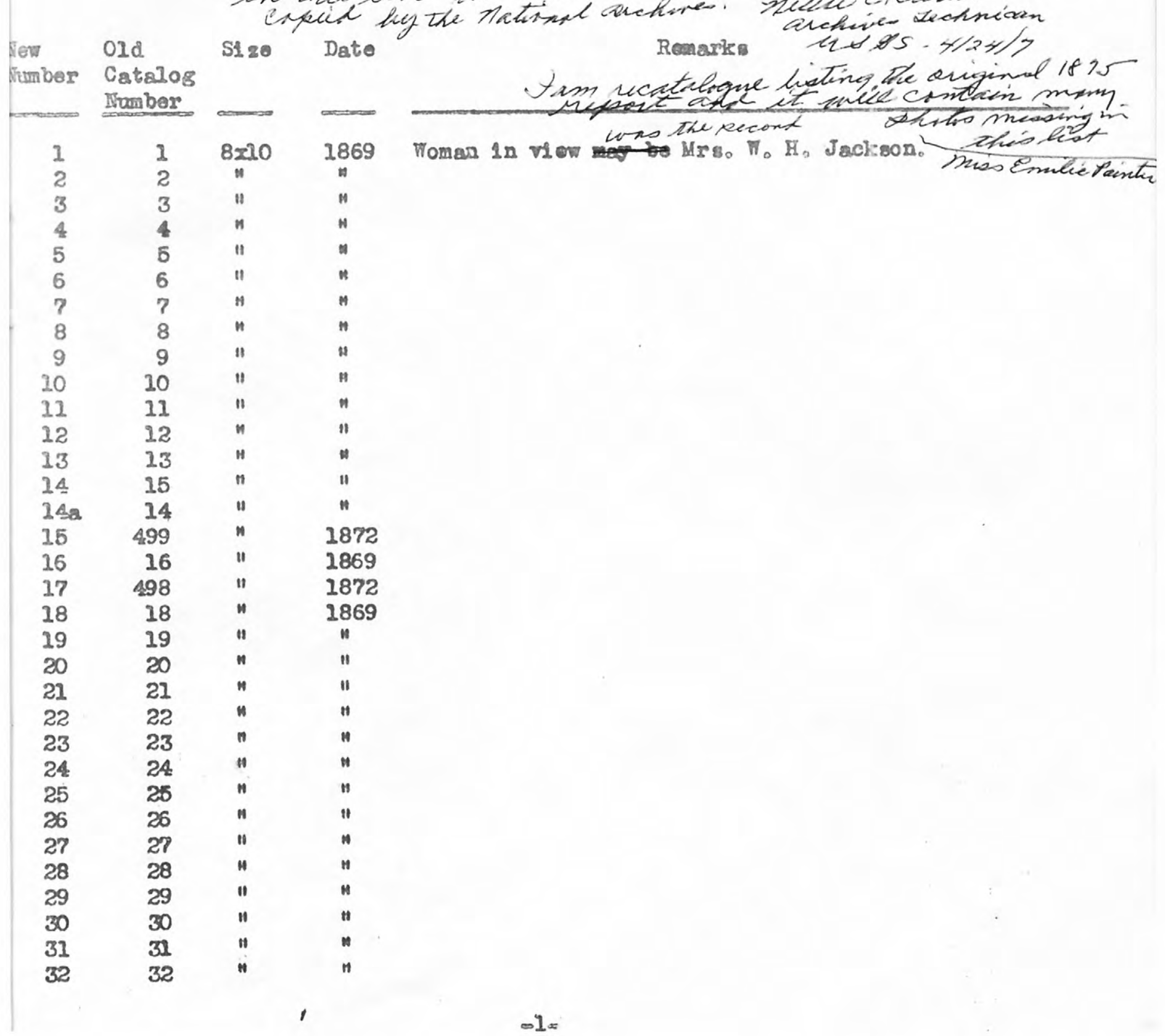




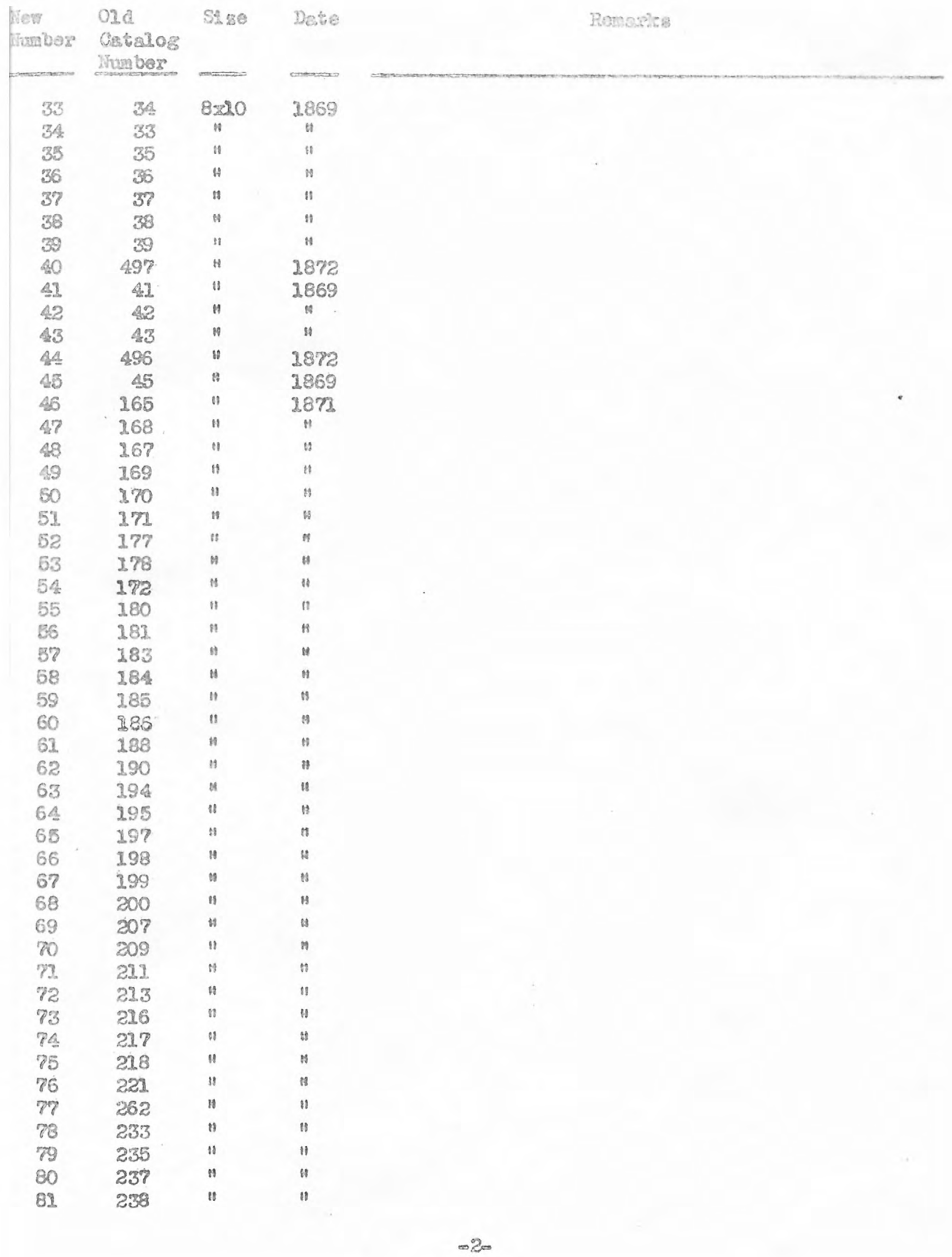




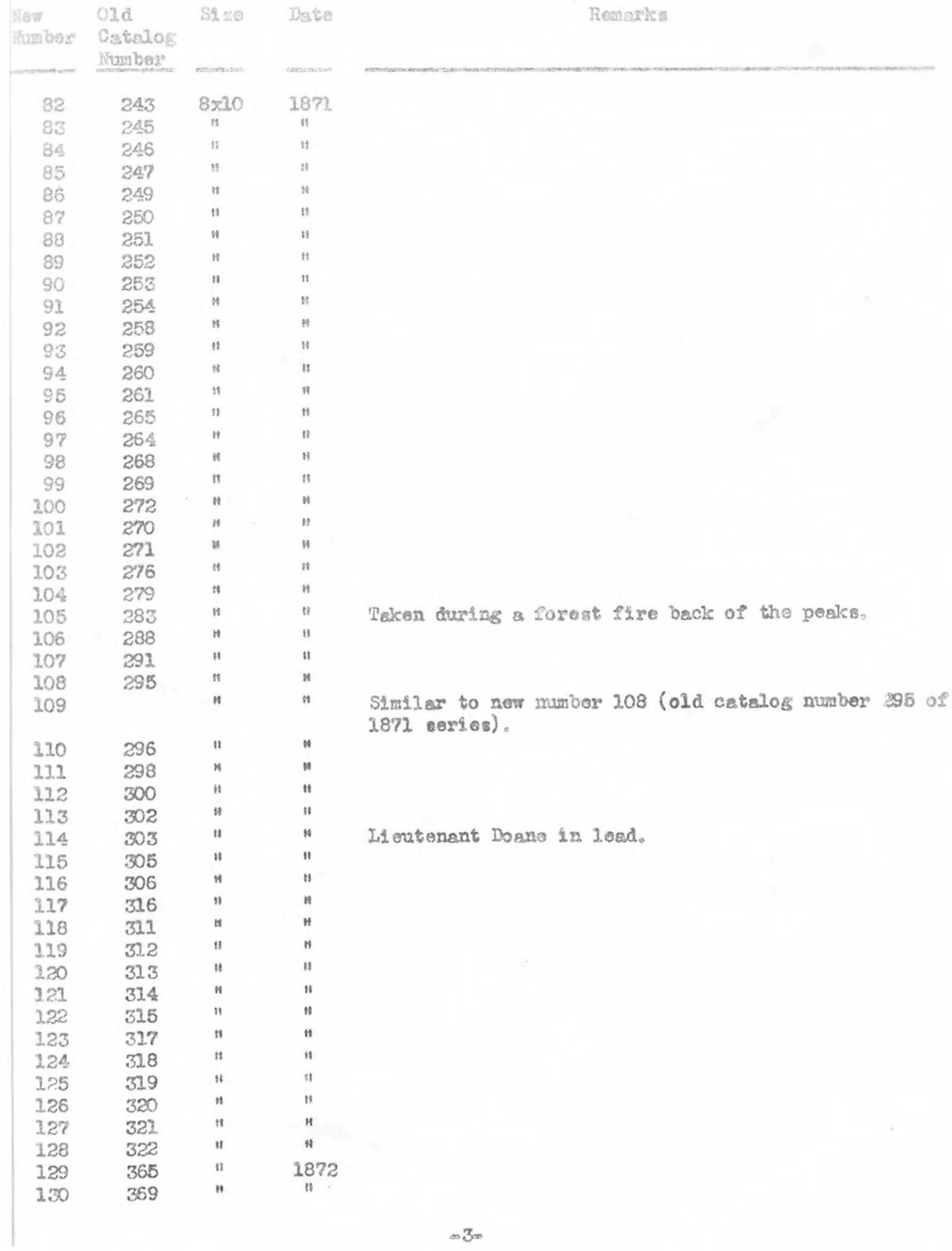




\begin{tabular}{|c|c|c|c|c|}
\hline $\begin{array}{l}\text { Now } \\
\text { Nomber }\end{array}$ & $\begin{array}{l}\text { Old } \\
\text { Catalog } \\
\text { Jwabor }\end{array}$ & ระ. & Das: & Resarks \\
\hline 131 & 372 & 8 selo & 1872 & \\
\hline 132 & 392 & * & & \\
\hline 133 & 373 & " & $\omega$ & \\
\hline 134 & 375 & $n$ & $\omega$ & \\
\hline 135 & 376 & " & " & \\
\hline 136 & 378 & $w$ & $n$ & \\
\hline 137 & 378 & " & $n$ & \\
\hline 238 & 379 & $"$ & $n$ & \\
\hline 139 & 381 & * & ต & \\
\hline 240 & 382 & $\omega$ & $n$ & \\
\hline 241 & 383 & ต & n & \\
\hline 142 & 384 & ต & $n$ & \\
\hline 143 & 385 & $n$ & N & \\
\hline 148 & 386 & H & n & \\
\hline 146 & 387 & $n$ & ต & \\
\hline 146 & 388 & " & " & \\
\hline 147 & 389 & $\omega$ & ต & \\
\hline 148 & 390 & $n$ & $\omega$ & \\
\hline 149 & 393 & " & $n$ & \\
\hline 180 & 394 & ต & " & \\
\hline 251 & 398 & n & " & \\
\hline 152 & 396 & 11 & $\omega$ & \\
\hline 183 & 397 & $n$ & " & \\
\hline 154 & 398 & 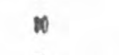 & $n$ & \\
\hline 156 & 399 & $"$ & n & \\
\hline 156 & 500 & ต & n & \\
\hline 157 & 101 & 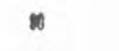 & $\omega$ & \\
\hline 158 & 402 & n & " & \\
\hline 189 & 103 & " & $n$ & \\
\hline 160 & 404 & " & " & \\
\hline 161 & 408 & " & $*$ & \\
\hline 162 & 109 & " & n & \\
\hline 162 & & " & " & 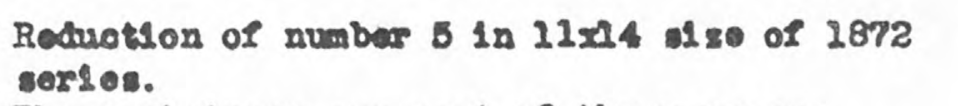 \\
\hline 163 & $412 P$ & $\omega$ & () & These plotures are part of the panorass \\
\hline 164 & 4231 & " & $\infty$ ) & numbersd 410 -414 (oId mubera) but thero 1 . \\
\hline 168 & 4147 & n & \% & $\begin{array}{l}\text { wow doubt that the old mubers assigned hero } \\
\text { are the comreot ones. }\end{array}$ \\
\hline 166 & 415 & $n$ & $n$ & \\
\hline 167 & 416 & $n$ & w & \\
\hline 168 & 428 & $n$ & n & \\
\hline 169 & 417 & $n$ & $n$ & \\
\hline 120 & 420 & $n$ & " & \\
\hline 17 & 422 & n & " & \\
\hline 172 & 423 & $n$ & n & $\begin{array}{l}\text { W. H. Jackson and Charles } R_{0} \text { Campell. HLgh } \\
\text { ledge comanding } \mathrm{Vlom} \text { of Tetons. }\end{array}$ \\
\hline 173 & 424 & " & $"$ & 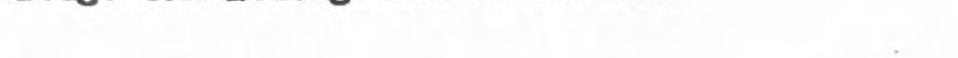 \\
\hline 174 & 435 & $w$ & $n$ & \\
\hline 175 & 426 & " & $n$ & \\
\hline 176 & 427 & n & n & \\
\hline 177 & 428 & $"$ & $"$ & \\
\hline
\end{tabular}




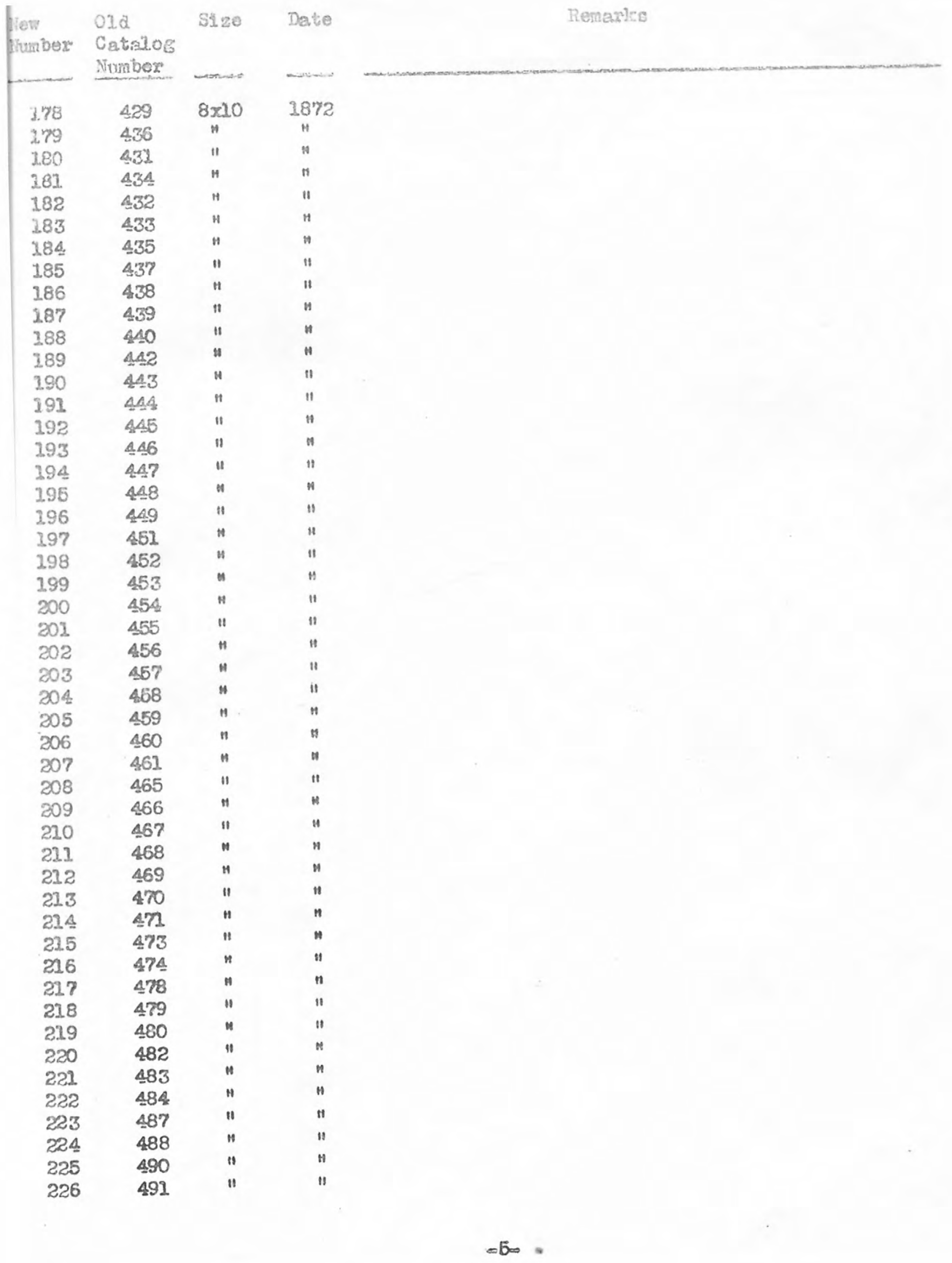




\begin{tabular}{|c|c|c|c|c|}
\hline $\begin{array}{l}\text { New } \\
\text { Number }\end{array}$ & $\begin{array}{l}\text { old } \\
\text { Catalog } \\
\text { Number }\end{array}$ & S2: $: 20$ & Dsce & Remarics \\
\hline 227 & 494 & $8 \times 230$ & 1872 & \\
\hline 228 & 493 & N & $H$ & \\
\hline 229 & 492 & $" 1$ & " & \\
\hline 230 & 495 & $"$ & $"$ & \\
\hline 231 & & $722.0 \frac{6}{6}$ & $1883 ?$ & $\begin{array}{l}\text { Crater of the Groat Fountaln Geysar. Pellowstone } \\
\text { Natlonal Park. }\end{array}$ \\
\hline 232 & & 72212 & $1883 ?$ & $\begin{array}{l}\text { Crater of the 01d Falthiul Geyser. Yollowatone } \\
\text { Natlonal Park. }\end{array}$ \\
\hline 233 & & $"$ & $2883 ?$ & $\begin{array}{l}\text { Crater of the Castle Geyser. Yellowstone National. } \\
\text { Park. }\end{array}$ \\
\hline 238 & & " & $1883 ?$ & Fountata Gaygar. Yellowakon Hational Park. \\
\hline 235 & & $n$ & $1883 ?$ & $\begin{array}{l}\text { Crosted Hot Springs. Old Fasthirl in the distance. } \\
\text { Yellowstono Natlonal Park. }\end{array}$ \\
\hline 236 & & $7 z 20$ & $1883 ?$ & Ione Star Geysor. Yellowstone Natlonal Park. \\
\hline 237 & & $7 \times 11$ & $2883 ?$ & Grotto Geyser. Yollowstono Nattonal Park. \\
\hline 238 & & " & 1883? & The Glantoss Gersor. Yellowgtone National Park. \\
\hline 239 & & " & $1883 ?$ & $\begin{array}{l}\text { Crasted Hot Sprines and Castlo Geyser. Yollowstono } \\
\text { Nationar Pari. }\end{array}$ \\
\hline 240 & & $8 \times 10$ & $1883 ?$ & IAttle Fire Hole Falls. Yellowntone Natlonal Park. \\
\hline 241. & & $7 \times 31$ & $1883 ?$ & $\begin{array}{l}\text { Md dway Gogser Basin. Crater of the Excelsior. } \\
\text { Yellowstone Hational Park. }\end{array}$ \\
\hline 242 & & " & $1883 ?$ & $\begin{array}{l}\text { Crater of the Castle Geyser. Yollomstone Natsonal } \\
\text { Park. }\end{array}$ \\
\hline 243 & & 6 줄 288 줄 & $1883 ?$ & 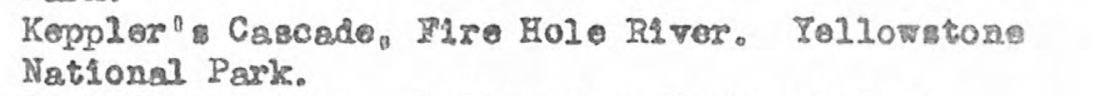 \\
\hline 240 & & $8 \times 10$ & $1883 ?$ & Lone Star Geyar. Yellowitone Rational Park. \\
\hline 245 & & $6 \frac{1}{2} 288 \frac{1}{2}$ & 1870 & $\begin{array}{l}\text { Hear duplicate of net mumber } 25 \% \text {. So romarks } \\
\text { sollowlag muber } 257 \text {. }\end{array}$ \\
\hline 216 & & $"$ & H & 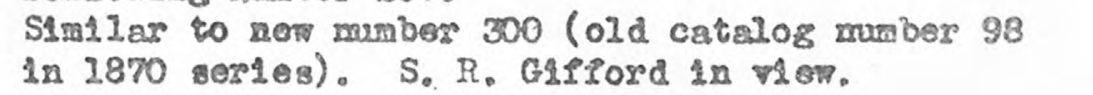 \\
\hline 247 & $4 ? ?$ & n & 18709 & \\
\hline 248 & 56 & n & 1870 & \\
\hline 249 & $\Delta 9$ & n & n & \\
\hline 250 & & n & a & 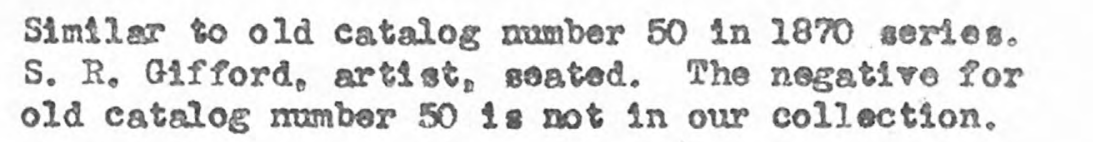 \\
\hline 251 & 51 & n & " & \\
\hline 252 & 52 & 11 & " & \\
\hline 253 & 53 & $n$ & $"$ & \\
\hline 254 & 54 & 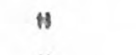 & " & \\
\hline 255 & 55 & $"$ & n & \\
\hline 256 & 68 & " & $"$ & \\
\hline 257 & 61 & 11 & $n$ & 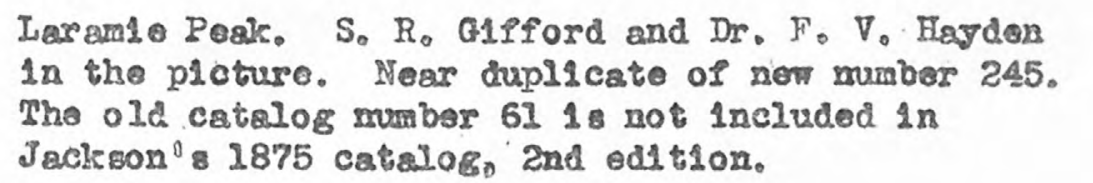 \\
\hline 258 & 59 & $"$ & a & \\
\hline 259 & 58 & $"$ & $n$ & \\
\hline 260 & 57 & $n$ & $"$ & \\
\hline
\end{tabular}




\begin{tabular}{|c|c|c|c|c|}
\hline Now & $\begin{array}{l}\text { Odd } \\
\text { Catalos } \\
\text { Iuspers }\end{array}$ & $5 \pm z e$ & Dateo & Henaries \\
\hline 261 & 60 & $6 \frac{2}{2} \times 8 \frac{2}{5}$ & 2870 & \\
\hline 262 & 62 & iี & n & \\
\hline 263 & 63 & n & $" 1$ & \\
\hline 264 & 64 & $n$ & " & \\
\hline 265 & 65 & $\omega$ & $\approx$ & S. $R_{0}$ Gerford in riow. \\
\hline 266 & 66 & $n$ & H & \\
\hline 267 & 67 & $n$ & $"$ & \\
\hline 258 & & $n$ & $n$ & Th1: plcture may have boen copled by Jackson. \\
\hline 269 & 69 & n & 10 & Contributed nogstive = not made by the Haydon Surveg. \\
\hline 270 & 20 & " & H & \\
\hline 272 & 71 & n & n & \\
\hline 272 & 72 & $n$ & : & \\
\hline 273 & 73 & n & n & \\
\hline 274 & 74 & n & $"$ & \\
\hline 275 & 75 & " & H & \\
\hline 276 & & $n$ & 1870 & 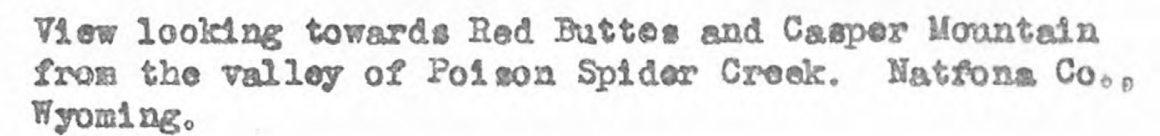 \\
\hline 277 & 76 & $n$ & 2870 & \\
\hline 278 & 77 & " & n & \\
\hline 279 & 78 & $n$ & " & \\
\hline 280 & & $n$ & n & $\begin{array}{l}\text { S1m lar to new number } 31.2_{0} \text { (old catalog muber } 116 \\
\text { In } 1870 \text { merten). }\end{array}$ \\
\hline 281 & 179 & "1 & n & \\
\hline 282 & 80 & $n$ & " & 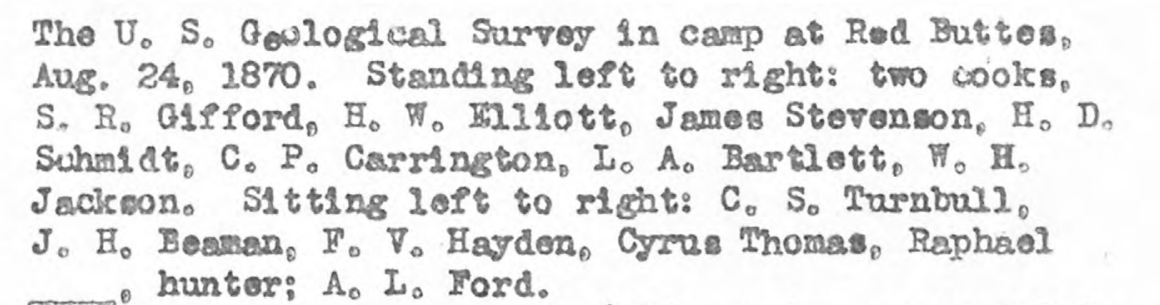 \\
\hline 283 & & 10 & " & 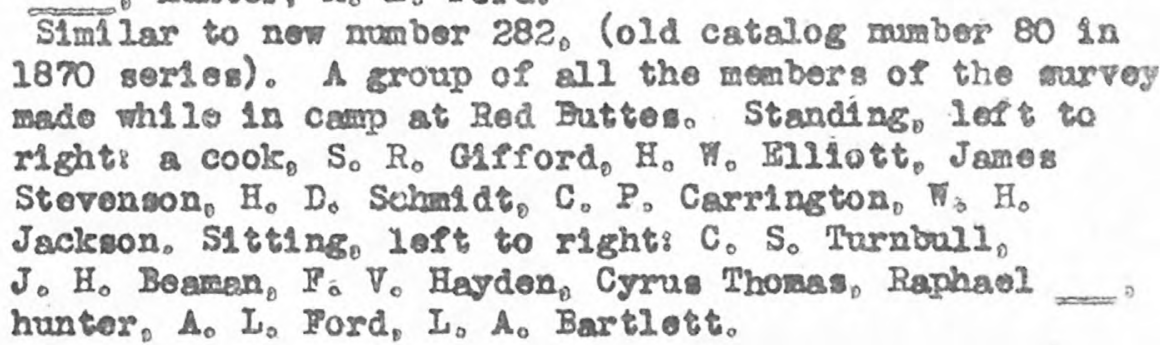 \\
\hline 284 & 81 & $n$ & $H$ & 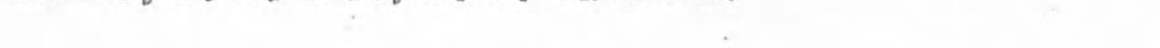 \\
\hline 286 & 83 & $n$ & 11 & \\
\hline 286 & 82 & n & " & 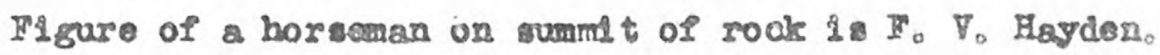 \\
\hline 287 & 85 & $n$ & " & \\
\hline 288 & 86 & $n$ & $"$ & $\begin{array}{l}\text { G1ford and Flliot in the foreground. Ambalance below } \\
\text { carrying photo working outlit. }\end{array}$ \\
\hline 289 & 87 & $n$ & H & \\
\hline 290 & 88 & $n$ & n & \\
\hline 291 & 89 & is & $n$ & \\
\hline 292 & 90 & $n$ & $"$ & \\
\hline
\end{tabular}




\begin{tabular}{|c|c|c|c|c|}
\hline $\begin{array}{l}\text { Naw } \\
\text { Nowber }\end{array}$ & $\begin{array}{l}\text { Old } \\
\text { Cacalos } \\
\text { Iusabor }\end{array}$ & $\$ 120$ & Date & Reverks \\
\hline 293 & 91 & $6 \frac{3}{2} 2 \times 8 \frac{2}{2}$ & 1870 & \\
\hline 294 & 92 & $\pi$ & n & \\
\hline 295 & 93 & $n$ & $n$ & \\
\hline 296 & 94 & $n$ & $n$ & \\
\hline 297 & 98 & H & $n$ & \\
\hline 298 & 96 & $"$ & $n$ & \\
\hline 299 & 97 & $"$ & " & \\
\hline 300 & 98 & N & $n$ & So $R_{0}$ Glfford its V1ew. Compare with new manbar 246. \\
\hline 301 & 100 & $n$ & $"$ & \\
\hline अ2 & 99 & " & n & \\
\hline 303 & 101 & " & " & \\
\hline 304 & & " & " & 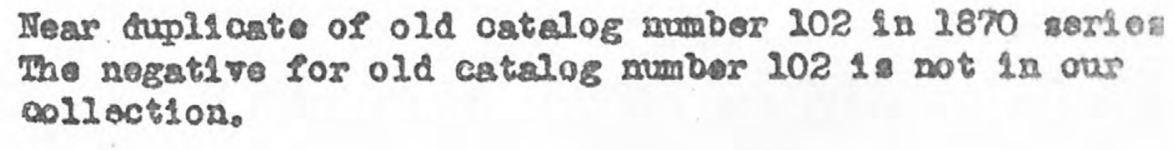 \\
\hline 306 & 207 & n & $"$ & 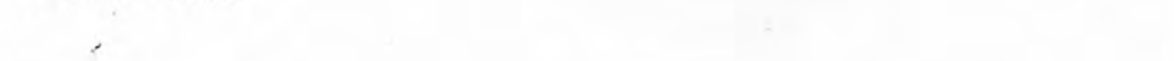 \\
\hline 306 & 210 & $n$ & $"$ & ${ }^{\prime}$ \\
\hline 507 & 211 & и & " & \\
\hline 308 & 112 & $"$ & " & \\
\hline 309 & 113 & $"$ & " & \\
\hline a10 & & u & N & $\begin{array}{l}\text { S1mi lar to now number } 323 \text { (old cstalos nubor } 127 \text { in } \\
18 \% \text { sortes). }\end{array}$ \\
\hline 31 & 115 & $n$ & n & \\
\hline 312 & 216 & $n$ & $"$ & S1m1lar to now number 280. \\
\hline 313 & 117 & n & $" 1$ & \\
\hline 314 & 218 & n & " & Iegat1vo destroyed. \\
\hline 316 & 219 & $"$ & $n$ & - \\
\hline 326 & 220 & $n$ & H & \\
\hline 31.7 & 121 & $n$ & $" 1$ & \\
\hline 31.8 & 122 & $" 1$ & $" 1$ & \\
\hline 319 & 123 & n & " & \\
\hline 320 & 124 & $"$ & $n$ & \\
\hline 321 & 125 & $n$ & $"$ & \\
\hline 322 & 226 & n & $n$ & \\
\hline 323 & 127 & $"$ & $n$ & \\
\hline 324 & 128 & $"$ & " & H. T. HIIlott in V1 OW. \\
\hline 325 & 129 & $"$ & n & 0 \\
\hline 326 & 130 & $n$ & $n$ & $\therefore$ \\
\hline 327 & 131 & $"$ & " & $\cdots$ \\
\hline 328 & 133 & " & " & . \\
\hline 329 & 136 & $"$ & n & \\
\hline 330 & 137 & n & $n$ & \\
\hline 331. & 138 & n & $\|$ & \\
\hline 332 & 139 & $"$ & m & \\
\hline 333 & 141 & $n$ & $n$ & \\
\hline 334 & 142 & $" 1$ & $n$ & $\cdot$ \\
\hline 335 & 143 & $\omega$ & $n$ & \\
\hline 336 & 144 & $n$ & $\omega$ & \\
\hline 337 & 146 & iv & " & \\
\hline 338 & 148 & " & $n$ & \\
\hline
\end{tabular}




\begin{tabular}{|c|c|c|c|c|}
\hline $\begin{array}{l}\text { Mew } \\
\text { Rumbor }\end{array}$ & $\begin{array}{l}\text { O1d } \\
\text { Cabarog } \\
\text { Ltrabar }\end{array}$ & S2 20 & Date & Remerk. \\
\hline 339 & 249 & $6 \frac{1}{2} \times \theta^{3}$ & 2870 & \\
\hline 30 & 160 & की & n & \\
\hline 341 & 151 & $"$ & $n$ & \\
\hline 342 & 153 & $n$ & $n$ & \\
\hline 343 & 185 & $n$ & "1 & \\
\hline $3 * 4$ & 2.56 & $n$ & $n$ & \\
\hline $3 A 6$ & 158 & " & $"$ & \\
\hline 346 & 159 & " & $n$ & \\
\hline $3 A 7$ & 160 & $n$ & " & \\
\hline 348 & 261 & $u$ & w & \\
\hline 349 & 257 & $5 \times 8$ & 1875 & \\
\hline 350 & & $"$ & & V2 ev of uniwown 1ocation. \\
\hline 361 & & $"$ & & V1ow of valenow Iocet1on. \\
\hline 352 & & " & 2878 & 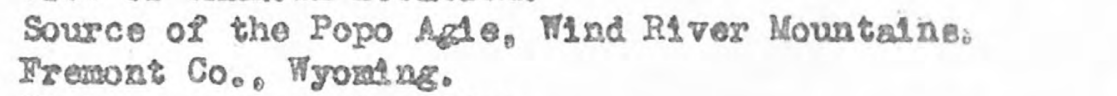 \\
\hline 353 & & " & & V1en of rnknown locat2on. \\
\hline 354 & & $n$ & & 01d Ta1thrvil Coyser. Tollowgtono NatLonal Paxk. \\
\hline 355 & & n & 2878 & 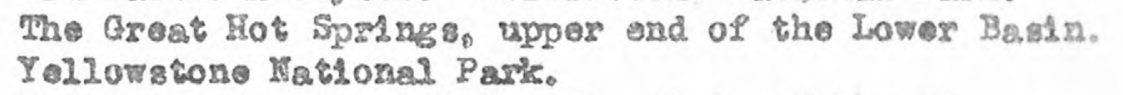 \\
\hline 356 & & " & " & 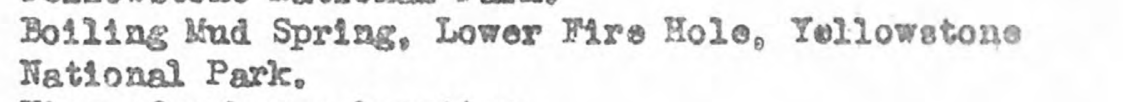 \\
\hline $35 ?$ & & n. & & V1 ow of wniknowa location. \\
\hline 358 & & $"$ & & Juscoz, Mextco. \\
\hline 369 & $x+7$ & n & 1874 & 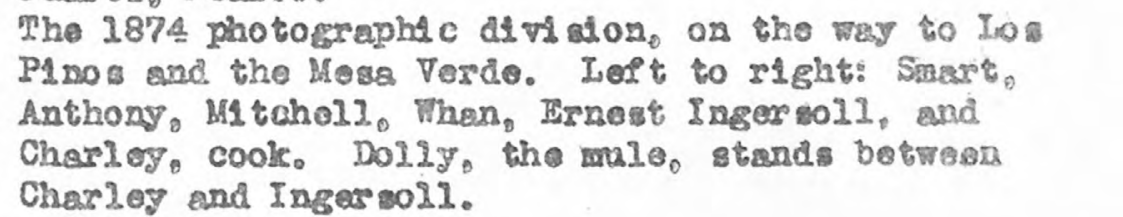 \\
\hline 360 & 2 & $"$ & 2873 & \\
\hline 361 & 3 & H & N & \\
\hline 362 & & " & 2878 & $\begin{array}{l}\text { Mamoth Hot Springs, the Frugan Watexiall. Yallow- } \\
\text { Btowe Natlonal Park. }\end{array}$ \\
\hline 363 & $6 ?$ & " & 1873 & \\
\hline 364 & 69 & $"$ & n & \\
\hline 365 & 7 & n & n & \\
\hline 366 & 8 & " & ท & \\
\hline 367 & & $n$ & $1878 ?$ & Towor Fa1. Yollowstone Nationol Park. \\
\hline 368 & 9 & н & 1873 & \\
\hline 369 & 10 & $n$ & n & \\
\hline 370 & 11 & m & " & \\
\hline 372 & 12 & $n$ & * & \\
\hline 372 & 13 & : & $"$ & \\
\hline 373 & & " & $1878 ?$ & Fail a of the Yellow tone. \\
\hline 374 & 18. & " & 1873 & \\
\hline 375 & 15 & " & $n$ & \\
\hline 376 & 16 & n & $n$ & \\
\hline 377 & 17 & n & $n$ & \\
\hline 378 & & $"$ & & Cascade Crook. Yollowstone Natlonsl Park, \\
\hline 379 & 19 & w & $n$ & \\
\hline 380 & 20 & $n$ & " & \\
\hline
\end{tabular}




\begin{tabular}{|c|c|c|c|c|}
\hline $\begin{array}{l}\text { Nen } \\
\text { Number }\end{array}$ & $\begin{array}{l}\text { Old } \\
\text { Catalos } \\
\text { Nherabs }\end{array}$ & Size & Deţe & Remarks \\
\hline 381 & 21 & $5 x 8$ & 1873 & \\
\hline 382 & & a & & VIow of the Haydon Vallog. Tollowstono Natlonal Parl \\
\hline 383 & 22 & " & 2873 & \\
\hline 384 & 23 & " & $" 1$ & \\
\hline 385 & 24 & H & 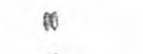 & \\
\hline 386 & 25 & $n$ & " & \\
\hline $38 \%$ & 26 & $"$ & " & \\
\hline 388 & 27 & $"$ & w & \\
\hline 389 & 28 & n & is & \\
\hline 390 & 29 & $n$ & $n$ & \\
\hline 392 & & " & $2878 ?$ & Bey wer in Yellowstono Tationel Park. \\
\hline 392 & 50 & " & 1873 & \\
\hline 393 & 31. & $" 1$ & 11 & \\
\hline 394 & 32 & $"$ & n & \\
\hline 395 & & " & $2878 ?$ & $\begin{array}{l}\text { Crater of the Great Fountaln Geyser. TolJowstone } \\
\text { Hational Park. }\end{array}$ \\
\hline 396 & 34 & 18 & 1873 & \\
\hline 397 & 33 & " & $" 1$ & Hogat1v・ m1 ร1ng. \\
\hline 398 & $130 ?$ & " & $187 \mathrm{~A}$ & \\
\hline 399 & 35 & $\|$ & 1873 & \\
\hline 400 & & " & $2878 ?$ & Excol stor Grymer. Yellowatone Hatlonal Paxis. \\
\hline 401 & 36 & $n$ & 3873 & \\
\hline 402 & 37 & n & " & \\
\hline 403 & 38 & $n$ & $\|$ & \\
\hline 804 & 39 & $"$ & $n$ & \\
\hline 405 & 40 & $"$ & $n$ & \\
\hline 806 & 48 & "1 & " & \\
\hline 407 & 42 & $"$ & $"$ & \\
\hline$\$ 08$ & 83 & $"$ & n & \\
\hline 809 & $4 A$ & $"$ & " & Negat1ve mselng. \\
\hline 490 & 45 & $"$ & ts & \\
\hline 481 & & $"$ & $1878 ?$ & 01d Fa1thrul Geysex. Yallovstono Nat1onal Park. \\
\hline 232 & 26 & $n$ & 2873 & \\
\hline 413 & 47 & n & n & , \\
\hline 42.4 & $\Delta 8$ & $n$ & n & • \\
\hline 235 & 49 & n & " & \\
\hline 416 & 50 & n & $a$ & \\
\hline 427 & 51 & $\omega$ & $n$ & \\
\hline 418 & 52 & $n$ & $n$ & \\
\hline 429 & 53 & $"$ & $n$ & \\
\hline 420 & 58 & $"$ & $n$ & \\
\hline 421 & 55 & $" 1$ & $n$ & Nogative deatroyed. \\
\hline 222 & 56 & $n$ & $"$ & \\
\hline 423 & 57 & is & $n$ & \\
\hline 424 & & $n$ & 28789 & $\begin{array}{l}\text { Blacir Sard Pool. upper basta of the Yollowstone. } \\
\text { Tollowstone Nations? Park. }\end{array}$ \\
\hline 425 & 58 & $n$ & 2873 & \\
\hline 425 & 39 & " & 11 & \\
\hline
\end{tabular}




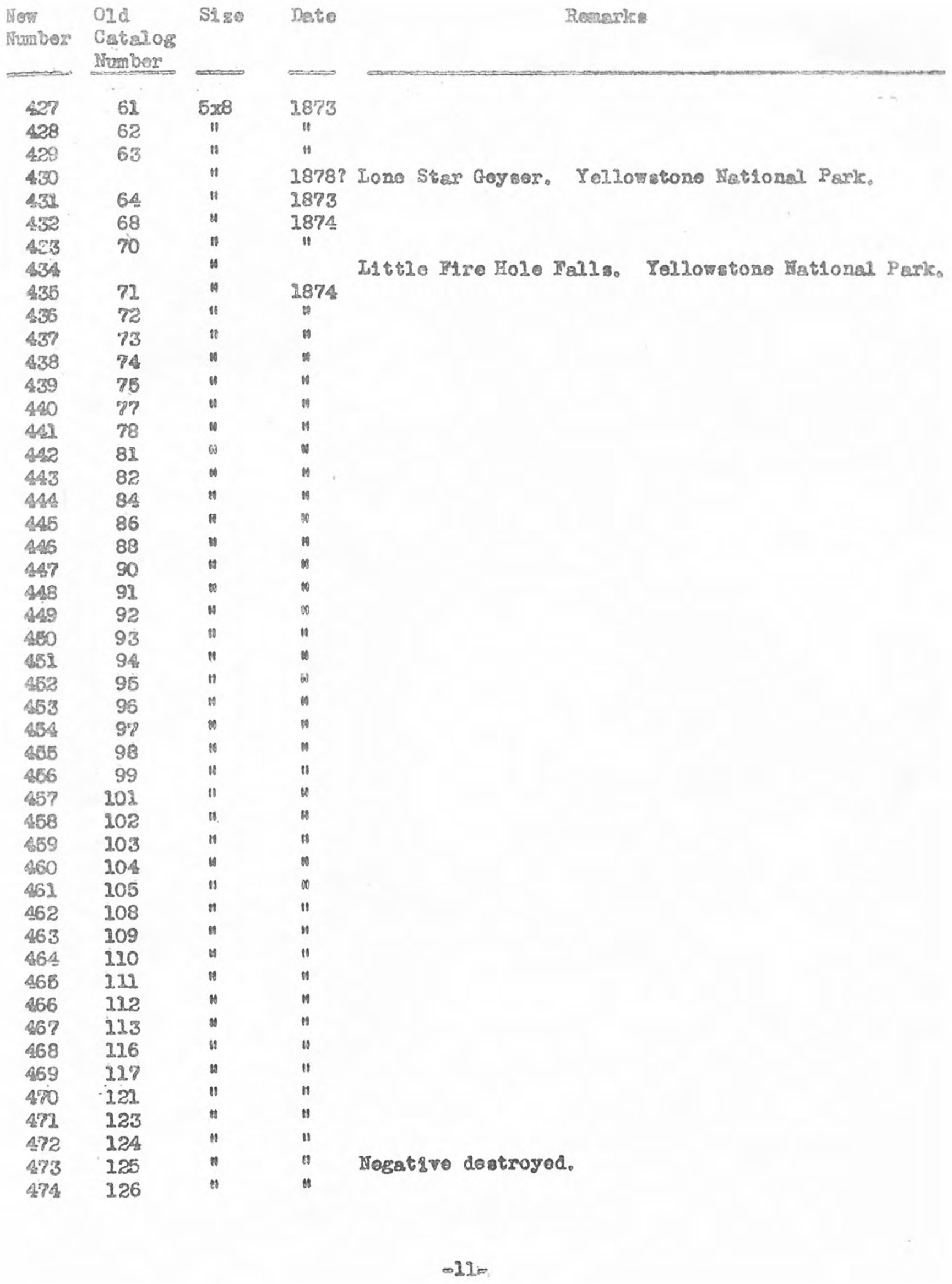




\begin{tabular}{|c|c|c|c|c|}
\hline $\begin{array}{l}\text { Rew } \\
\text { NhmDos }\end{array}$ & $\begin{array}{l}\text { O1d } \\
\text { Catajog } \\
\text { Inombers }\end{array}$ & Si 80 & Dato & Rewarkg \\
\hline 475 & -129 & $5 \times 8$ & 1874 & \\
\hline 476 & $\Delta$ & " & 1873 & \\
\hline 477 & $232 ?$ & n & $187 \%$ & \\
\hline 478 & 133 & $\leftrightarrow$ & n & \\
\hline 479 & 134 & $6 \frac{7}{2} 28 \frac{1}{2}$ & 2870 & Th1 negatito has beon cut down to $5 x 8$. \\
\hline 480 & 134 & $5 \pi 8$ & 2878 & \\
\hline 481 & 135 & " & " & \\
\hline 482 & 138 & " & " & \\
\hline 883 & 139 & $n$ & $n$ & . \\
\hline 484 & 340 & " & $"$ & \\
\hline 485 & 212 & " & $"$ & \\
\hline A86 & 142 & " & H & \\
\hline 287 & 143 & $n$ & n & \\
\hline 488 & 242 & н & n & \\
\hline 489 & & n & & Hs gure 2 \% Janos Stophenson. \\
\hline 490 & & $n$ & $28793 \%$ & 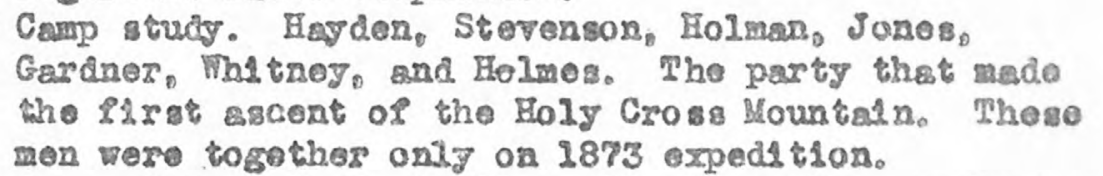 \\
\hline 491 & & $"$ & $1873 ?$ & 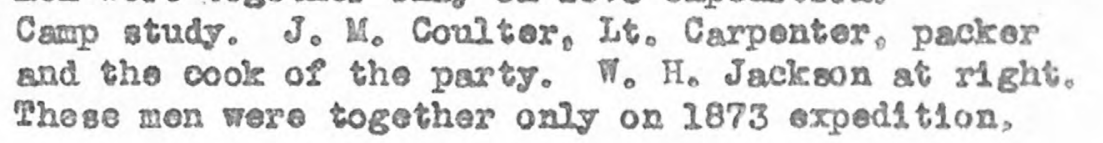 \\
\hline 492 & 146 & $n$ & 1874 & \\
\hline$\Delta 93$ & 145 & $"$ & " & \\
\hline 494 & 147 & и & "I & \\
\hline 496 & 148 & $n$ & $n$ & \\
\hline 496 & 149 & 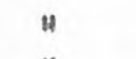 & $n$ & \\
\hline 497 & 150 & $"$ & $"$ & \\
\hline 498 & 151 & " & w & Megatsvo deztroyed. \\
\hline 499 & . & " & $\begin{array}{l}1871 ? \\
1872 ?\end{array}$ & 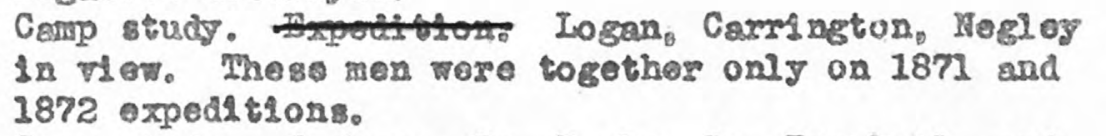 \\
\hline 500 & & $n$ & 18719 & 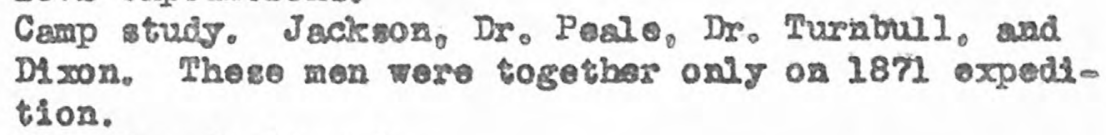 \\
\hline 302 & 184 & " & 1878 & Hegat1vo destroyed. \\
\hline 502 & 183 & w & n & Goolog1 ๘6 ล6 work. \\
\hline 503 & & $"$ & & Camp study, Easden and Walter Pard a. \\
\hline 504 & 255 & " & 1875 & \\
\hline 605 & 251 & $"$ & n & \\
\hline 506 & 381 & H & 1874 & 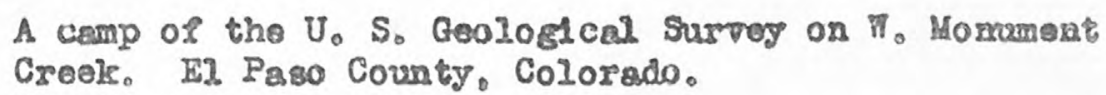 \\
\hline 507 & 180 & $"$ & " & Negative destroyed. \\
\hline 508 & 178 & n & $n$ & Pack animals visth "Aparojos" \\
\hline 509 & $2 A B$ & $n$ & 2875 & \\
\hline 520 & w & w & $1871 ?$ & $\begin{array}{l}\text { Snake River up Irom Taylor" Bridge. Nogative } \\
\text { destroged. }\end{array}$ \\
\hline 511 & $2 A 6$ & $"$ & 1875 & \\
\hline
\end{tabular}




\begin{tabular}{|c|c|c|c|c|}
\hline Then & $\begin{array}{l}\text { 01d } \\
\text { Catiolog } \\
\text { Numbor }\end{array}$ & Sมี & Date & Renaris: \\
\hline 512 & 276 & $5 \times 8$ & 2874 & 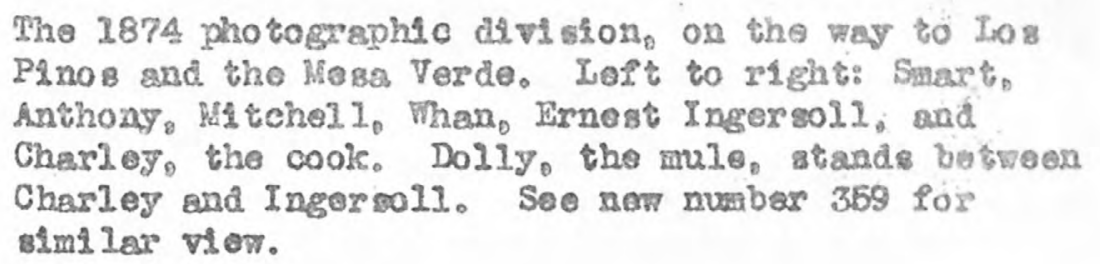 \\
\hline 513 & 242 & $n$ & 1875 & \\
\hline 514 & 1785 & $n$ & 2874 & Three very good Priends. \\
\hline 515 & $7 \%$ & $n$ & 11 & 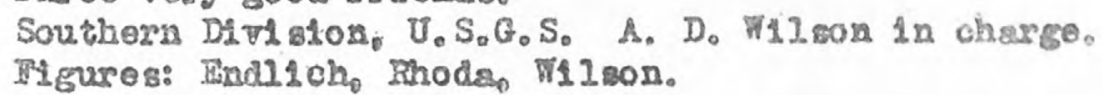 \\
\hline 516 & $122 ?$ & $"$ & s & \\
\hline 517 & 17 & $n$ & $n$ & \\
\hline 518 & & $n$ & 2870 & $\begin{array}{l}\text { Hamnoth Hot Springs, Iower basins, looklng rp. } \\
\text { Yollowetone Nat1ongl Paris. }\end{array}$ \\
\hline 529 & 170 & $"$ & 1878 & \\
\hline 520 & 169 & n & w & \\
\hline 521 & $.15 ?$ & $"$ & $"$ & W. H。 Holmes in V1 ow. \\
\hline 522 & 89 & $n$ & " & \\
\hline 523 & 83 & $"$ & " & \\
\hline 524 & $133 ?$ & " & $n$ & \\
\hline 525 & 79 & $"$ & $"$ & \\
\hline 526 & 65 & $"$ & $" 1$ & 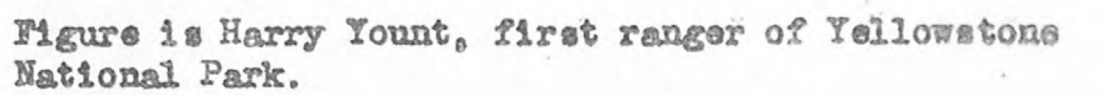 \\
\hline 527 & 60 & ต & 1873 & \\
\hline 528 & 155 & $" 1$ & 2874 & \\
\hline 529 & 164 & $n$ & " & \\
\hline 530 & & " & 1878 & $\begin{array}{l}\text { Cracer of the Castle Geymer. Yallombtore Nathoral } \\
\text { Paric. }\end{array}$ \\
\hline 633 & & n & 18789 & $\begin{array}{l}\text { Great Hot Spsing. near the Grand Gogser. Yeliow } \\
\text { wtone Hatlonel Pork. }\end{array}$ \\
\hline 532 & 293 & n & 2875 & \\
\hline 633 & 291 & " & & $\begin{array}{l}\text { Craters of the Grand and Turiban Gaysers. Tellow- } \\
\text { stone Natlonal Park. Nogat1ve st astrg. }\end{array}$ \\
\hline 534 & 287 & $"$ & 2875 & \\
\hline 535 & & $n$ & 2878 & $\begin{array}{l}\text { Crater of the Olant Geyer. Tollowntone Nathonal } \\
\text { Park. }\end{array}$ \\
\hline 538 & 279 & "1 & 2875 & 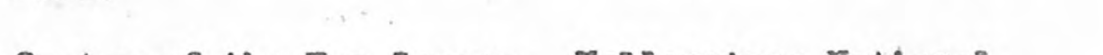 \\
\hline 557 & & " & 1878 & $\begin{array}{l}\text { Crater of the Fas Geyer. Tallowstane Natlonal } \\
\text { Park. }\end{array}$ \\
\hline 838 & $27 \%$ & w & 1875 & \\
\hline 539 & 276 & $n$ & w & \\
\hline 540 & & n & -0 & 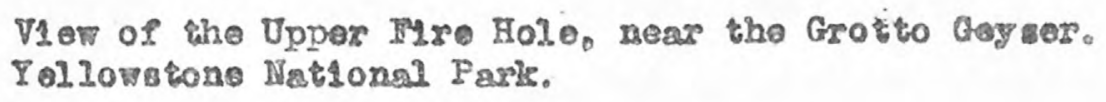 \\
\hline 542 & 274 & s & $18 \% 5$ & \\
\hline 582 & 273 & "1 & $"$ & \\
\hline 543 & & $"$ & 18789 & General Thew of IEgrta Springs. \\
\hline 644 & 372 & n. & 1878 & 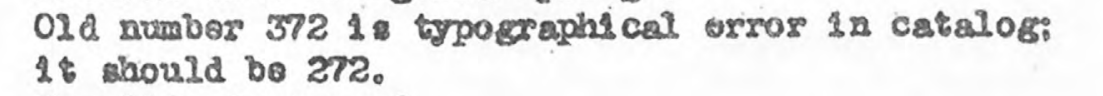 \\
\hline $5 \leq 5$ & 271 & $n$ & H & 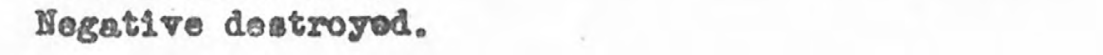 \\
\hline
\end{tabular}


$\begin{array}{ll}\text { New } & \text { 0ld } \\ \text { Number } & \text { Catraios }\end{array}$

Itum ber

\begin{tabular}{|c|c|c|c|}
\hline 546 & 270 & $5 \times 8$ & 1875 \\
\hline 547 & 269 & " & $"$ \\
\hline 548 & & $"$ & \\
\hline 149 & . & " & \\
\hline 550 & 268 & " & 1875 \\
\hline 551 & 267 & $" 1$ & " \\
\hline 552 & 266 & $"$ & $n$ \\
\hline 553 & 265 & 11 & $"$ \\
\hline 554 & 262 & " & $"$ \\
\hline 555 & 261 & $"$ & 11 \\
\hline 556 & 260 & $"$ & M \\
\hline $55 ?$ & 259 & " & " \\
\hline 58 & & n & 3878 \\
\hline
\end{tabular}

559

560

$56 ?$

562

563

564

565

366

567

568

569

570

572

572

573

574

575

576

57

578

\section{SI 20}

1875
$n$
$n$
11
$"$
11
$n$
11
1878

1875

1878

264. "

250

252

$24 A$

211

243
2875

1878 ?

1875

11

1878

M

1875

11

"

2878

$98 \% 3$

1878

Conch Spring Lower Fire Holo. Yollowstone Natsonal Parks.

The Is 1 berty $\mathrm{Cap}_{\mathrm{p}}$ Mamnoth Hot SprMngs。. Yellowetono National Parls.

The boaut1ful Bo111ng Spring. Lower Fise Hole.

Yellowtone Kational Paric.

Crater of the Forntala Coyser, Iower Fire Hole. Tollomgtone Natsonal Park.

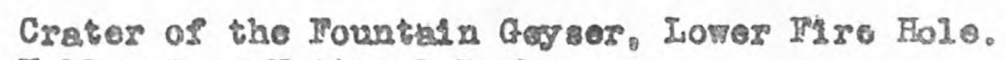
Yollowstono Natsonal Paxt.

D.stant view of Fourtsin Geyser, Lower Fro Hule. Yal lowitone Nationsl Park.

Whst Cone Geyser. Lomer F1re Hole. Yellowtono National Park.

Nagat1vo destroyed.

Posine of axtlnct sprinso Mamoth Hot Springs. Yellowstone Netional Parik.

Gian Spring, Mamoth los Springe. Fellowstone National Park.

Sand dunos, noer Mosca Pass, Colorado.

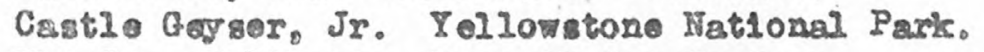
The Cap of ILborty. Mamoth Hot Springa. Yollorstone Nat1onal Park.

Tht 8 may not be a Iackson plroto. Unknown Iocall ty. Memoth Hot Springmo sumit of terrace. Yollowetone Ratlonal Park. 


\begin{tabular}{|c|c|c|c|c|}
\hline $\begin{array}{l}\text { Now } \\
\text { Irumiber }\end{array}$ & $\begin{array}{l}\text { Old } \\
\text { Cabalog } \\
\text { Tombers }\end{array}$ & Stze & Dete & Remarics \\
\hline 579 & & $5 x 8$ & 1878 & $\begin{array}{l}\text { Menmoth Hot Springs, aumit of first terrace. } \\
\text { Tratertine Fan. Iellowstone National Park. }\end{array}$ \\
\hline 580 & & $\Leftrightarrow$ & $u$ & $\begin{array}{l}\text { Crater of Fountan Geyser, Lower Fise Hole. } \\
\text { Yollowstone National Paxi. }\end{array}$ \\
\hline 581 & 229 & N & & $\begin{array}{l}\text { Heramoth Hot Springs, the Frozon Watarsall. Yollowe } \\
\text { stone National Park. Moget1ve destroyod. }\end{array}$ \\
\hline 582 & & n & & $\begin{array}{l}\text { Mannoth Hot Springs, near sumit of Plsit terrace. } \\
\text { Yellowitone Nat1onal Paric. }\end{array}$ \\
\hline 583 & & $"$ & & 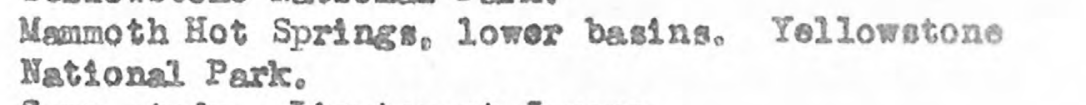 \\
\hline 584 & & $"$ & & Carp \&trdy. IL vtensnt Jerome. \\
\hline $\begin{array}{l}685 \\
586\end{array}$ & 215 & $"$ & $\begin{array}{l}187 \mathrm{~A} \\
2878 ?\end{array}$ & 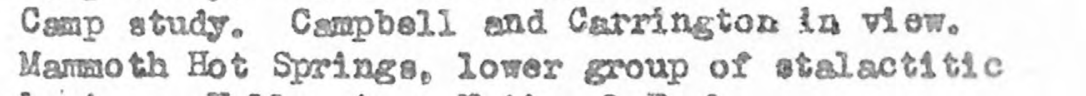 \\
\hline 587 & & $"$ & $\begin{array}{l}2872 ? \\
1872 ?\end{array}$ & 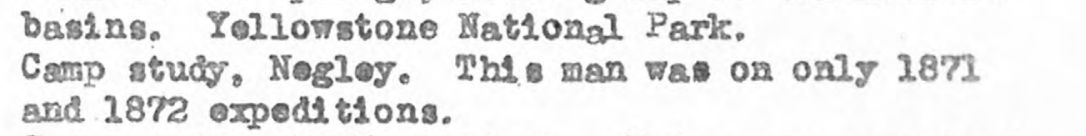 \\
\hline 588 & & n & $\begin{array}{l}1870 ? \\
1871 ?\end{array}$ & 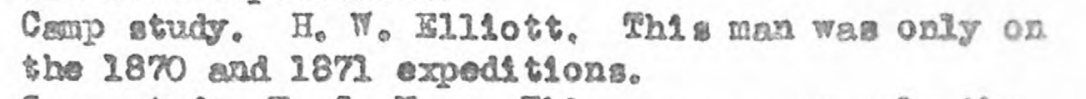 \\
\hline 589 & & $" 1$ & $2871 ?$ & 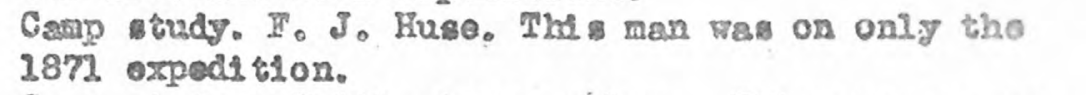 \\
\hline 690 & & $n$ & $1871 ?$ & 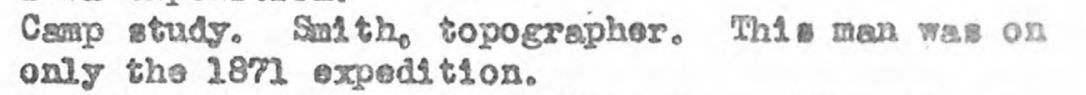 \\
\hline 591 & & $n$ & & $\begin{array}{l}\text { Manmoth Hot Springs, v1 ew Irom uppar terraco. } \\
\text { Tal10watone Nat1onal Park. }\end{array}$ \\
\hline 592 & 210 & $"$ & & Camp study. W. H. Jackron. Hegativo destroyod. \\
\hline 593 & & " & 2878 & 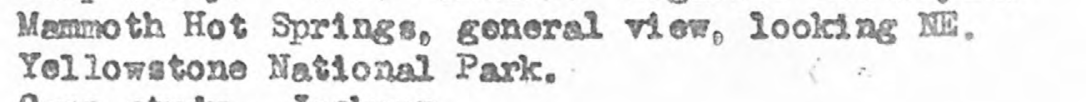 \\
\hline 594 & & $"$ & & Camp atrudy. Jackson. \\
\hline $\begin{array}{l}595 \\
596\end{array}$ & & " & & 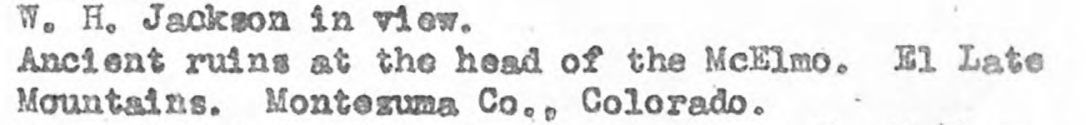 \\
\hline 598 & & w & 1878 & $\begin{array}{l}\text { Mamoth Hot Springs, looking north to the Yellowe } \\
\text { atone. Tallowstone Natlonal Park. }\end{array}$ \\
\hline 598 & & $"$ & 28719 & 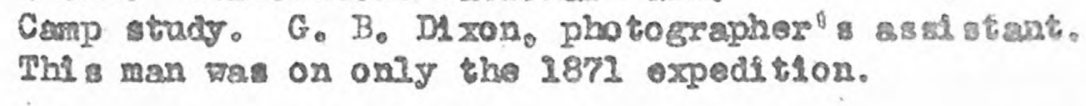 \\
\hline 599 & 295 & "I & 1875 & \\
\hline $\begin{array}{l}600 \\
501\end{array}$ & 160 & " & 1874 & Mamoth Hot Springs, looking north to the valley \\
\hline 602 & & "1 & & $\begin{array}{l}\text { of the Yollowstone. Yallowstone National Part. } \\
\text { Anctent sulns in the canyon of tho Manoos. } \\
\text { Colosado. }\end{array}$ \\
\hline 603 & 291. & is & 2875 & \\
\hline $60 \mathrm{~s}$ & & $"$ & $2878 ?$ & Togwoten Pess. Fyoming. \\
\hline $60 B$ & & n & $1878 ?$ & Mounc Leldy. Buffalo Fork. Innooln Co. FronIng. \\
\hline 606 & & $"$ & $2878 ?$ & Badlands on Find RI \\
\hline $\begin{array}{l}607 \\
609\end{array}$ & & $n$ & $\begin{array}{l}1878 \\
1878 ?\end{array}$ & V10\% Irom Togworos Pass. Incoln Co. Fyoming. \\
\hline & & $n$ & & Towar Fall. Yallowstone Naţonal Park. \\
\hline
\end{tabular}




\begin{tabular}{|c|c|c|c|c|}
\hline $\begin{array}{l}\text { Nem } \\
\text { Number }\end{array}$ & $\begin{array}{l}\text { OId } \\
\text { Catalog } \\
\text { lumber }\end{array}$ & Stze & Dare & Rssistes \\
\hline $\begin{array}{l}609 \\
610\end{array}$ & & $\underset{n}{6 \pi 8}$ & $\begin{array}{l}1878 ? \\
1878 ?\end{array}$ & $\begin{array}{l}\text { Wind River Peak. Fremont Co., Froming. } \\
\text { V1en north srom Fremonts Peak. Fremont Co. } \\
\text { Wyoming. }\end{array}$ \\
\hline 611 & & $"$ & 2.878 & $\begin{array}{l}\text { Heart Isire and Mt. Shexidara. Tellowstone } \\
\text { Batlonal Park. }\end{array}$ \\
\hline 612 & & $n$ & $1878 ?$ & $\begin{array}{l}\text { Sumnt of Fremont Peak. Fremont Co. } \\
\text { Fyoming. }\end{array}$ \\
\hline 613 & & $" 1$ & $1878 ?$ & $\begin{array}{l}\text { Glen Spriog, Hamath Bot Springn. Yollowstone } \\
\text { Natlonal Park. }\end{array}$ \\
\hline 614. & & n & 18789 & $\begin{array}{l}\text { Cratar of the Poarl Geyser, Haart Lako Bandn. } \\
\text { Yollowstone Mathonal Park. }\end{array}$ \\
\hline 615 & & ต & $2878 ?$ & $\begin{array}{l}\text { Crater of the Pearl Gegaes. Heart Lake Bastn. } \\
\text { Tal10\%atone Matsonal Park. }\end{array}$ \\
\hline 616 & & " & 18789 & $\begin{array}{l}\text { Pearl Goyger Cone, Hoart Iake Basin. } \\
\text { Yollowtone National Park. }\end{array}$ \\
\hline $\begin{array}{l}627 \\
618\end{array}$ & 258 & " & 1875 & Camp undes the Tetons, Iincoln Co. Wroming. \\
\hline 619 & 154 & $6 / 2 \times 81 / 2$ & 1870 & ThI negative has bsen cut down to $5 x 8$. \\
\hline 620 & & $5 \times B$ & 1878 & $\begin{array}{l}\text { Fissure Sprtags, Hoart loke Basin. } \\
\text { Yollowione National Park. }\end{array}$ \\
\hline 621 & & H & H & 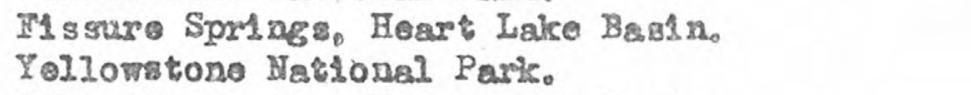 \\
\hline 622 & & $"$ & $"$ & $\begin{array}{l}\text { Columbia Spring, Hear L Inke Basin. } \\
\text { Yol Iowtono FatLonal Park. }\end{array}$ \\
\hline 623 & & " & n & $\begin{array}{l}\text { Crater of Inatic Garser. Hears Lake Basino } \\
\text { Yollowatone National Park. }\end{array}$ \\
\hline 624 & & 11 & " & $\begin{array}{l}\text { Crater of Rugtic Geysor, Heart Inire Basta. } \\
\text { Yeliowstono Nat1onal Park. }\end{array}$ \\
\hline 625 & & $n$ & $1878 ?$ & $\begin{array}{l}\text { Coral Spring, Shomhone Batn. Yollowntone } \\
\text { Natlonal Pari. }\end{array}$ \\
\hline 626 & & " & 1878 & $\begin{array}{l}\text { Corai Spring. Sho ahono Basin. Tel. lowstone } \\
\text { National Parie. }\end{array}$ \\
\hline 627 & & n & " & $\begin{array}{l}\text { Iallow Spongo Springs Sho ghone Bagtn. } \\
\text { Yellow sone Nat1onal Parle. }\end{array}$ \\
\hline 628 & & " & $1878 ?$ & $\begin{array}{l}\text { Yollow Sponge Spring, Sho abone Basln. } \\
\text { Yollowstono Natlonal Park. }\end{array}$ \\
\hline 629 & & is & $1878 ?$ & $\begin{array}{l}\text { Crater of the Inton Geyger, Sho shone Barin. } \\
\text { Yellowstons National Park. }\end{array}$ \\
\hline 630 & & " & 2878 & $\begin{array}{l}\text { Hht to Velves Spring, Sho shone Basin. } \\
\text { Yellowstors Natlonal Park. }\end{array}$ \\
\hline 631 & & n & " & $\begin{array}{l}\text { Brom Sponge Sprlng, Shoshone Babin. } \\
\text { Yellowatone Natlonal Park. }\end{array}$ \\
\hline 632 & & " & $1878 ?$ & $\begin{array}{l}\text { Whit to Velvet Spring, Sho ahono Bsaln. } \\
\text { Yollowstono Hatlonal Park. }\end{array}$ \\
\hline 633 & & " & 1878 & $\begin{array}{l}\text { Brouze Spring, Shosbono Basin. } \\
\text { Tellowstone Natlonal Parl. }\end{array}$ \\
\hline 634 & & $" 1$ & $1878 ?$ & $\begin{array}{l}\text { Bronze Spring, Sho shone Basln. } \\
\text { Yellowstono Natlonal Park. }\end{array}$ \\
\hline
\end{tabular}




\begin{tabular}{|c|c|c|c|c|}
\hline $\begin{array}{l}\text { Nov } \\
\text { Nunbor }\end{array}$ & $\begin{array}{l}\text { Old } \\
\text { Catalog } \\
\text { Neraber }\end{array}$ & Size & Dato & Romaxics \\
\hline 635 & & $5 \times 8$ & $3878 ?$ & $\begin{array}{l}\text { Genoral view in the Shoghone Geyser Bastn. } \\
\text { Yellowatone National Park. }\end{array}$ \\
\hline 636 & & n & $1878 ?$ & $\begin{array}{l}\text { Rosette Syring, Shoshone Bacin. Yellowstone } \\
\text { National Park. }\end{array}$ \\
\hline 637 & & n & 3878 & $\begin{array}{l}\text { Craters of the Gourd. Shield, and Mnuto Man, } \\
\text { Sho ghone Bastin. Yellowstone Mationsl Park. }\end{array}$ \\
\hline 638 & & $" 1$ & " & $\begin{array}{l}\text { Crater of the Minute Man, Sho ahone Bacin. } \\
\text { Pellovatone National Park. }\end{array}$ \\
\hline 639 & & i & & The Grest Totons。 IIncoln Co.0 Fyoming. \\
\hline 640 & & $n$ & 1878 & $\begin{array}{l}\text { The Leaser Mereild Pool, on Irom Spring Creole. } \\
\text { Yellowstoue Nationsl Park. }\end{array}$ \\
\hline 681 & & $"$ & t & 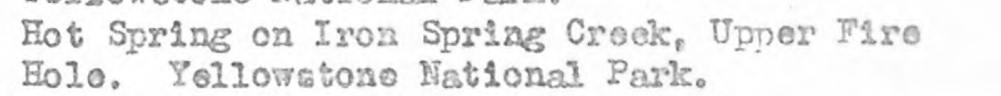 \\
\hline 642 & & $" 1$ & $H$ & $\begin{array}{l}\text { Merble Clip Spring, Shoshone Basin. } \\
\text { Tellowatone National Park. }\end{array}$ \\
\hline 643 & & $n$ & " & $\begin{array}{l}\text { Vian on Iron Spring Croek, Upper Fire Hole. } \\
\text { Yellowstone Natsonal Parik. }\end{array}$ \\
\hline 6A4 & 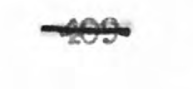 & $n$ & $\because$ & $\begin{array}{l}\text { VAow Prom the Black Sand Spring. Yollowstono } \\
\text { Netional Park. Hegativo destroyed. }\end{array}$ \\
\hline 645 & & $"$ & " & $\begin{array}{l}\text { Blacir Sand Springo Uppor Wire Fole. } \\
\text { Yollowacone National Park. }\end{array}$ \\
\hline 646 & & $"$ & $n$ & $\begin{array}{l}\text { Three Crater Spings Upper Fire Eole. } \\
\text { Yollowatoze Kational Park. }\end{array}$ \\
\hline 647 & & $"$ & $3878 ?$ & 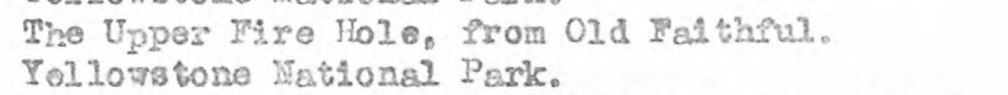 \\
\hline 648 & 370 & 82210 & 1872 & $\begin{array}{l}\text { The negative Por this number has been cut down } \\
\text { to } 5 \pi 8 \text {. }\end{array}$ \\
\hline 649 & 367 & $"$ & $"$ & \\
\hline $\begin{array}{l}650 \\
651\end{array}$ & 308 & $\begin{array}{l}6 x 8 \\
\text { stereo }\end{array}$ & 1875 & Crater of 01d Falthrus. Yellowstone Nattonal \\
\hline 652 & & n & & $\begin{array}{l}\text { In Lowor Gerser Bestr. Yellowstone Hational } \\
\text { Park. }\end{array}$ \\
\hline 653 & & $" 1$ & & Uppor Basin. Yellowatons National Park. \\
\hline 654 & & $"$ & & Uppor Basin. Yellowstono National Park. \\
\hline 655 & & $" 1$ & & $\begin{array}{l}\text { Saw Mi11 Garsar. Uppor Basin. Yellowstono } \\
\text { Nastonal Park. }\end{array}$ \\
\hline 656 & & $H$ & & $\begin{array}{l}\text { Upper Bastas tron neer Grand. Yellovatono } \\
\text { Natsonal Park. }\end{array}$ \\
\hline 657 & & $"$ & & Uppor Basin. Yollowatone Tational Perk. \\
\hline 658 & & $"$ & & Upper Bardn. Yellowatono Pational Park. \\
\hline 659 & & H & & $\begin{array}{l}\text { Sponge Geyser, Uppor Basln. Yollowstone } \\
\text { Natlonal Park. }\end{array}$ \\
\hline 660 & & $" 1$ & & 01d Falthrul. Yallonbtone Natsonal Park. \\
\hline 561 & & " & & Cast10 Gegser. Yellowgtone Natlonal Park. \\
\hline 562 & & H & & Lone Star Geyser. Yollowstone Natlonal Park. \\
\hline 653 & & 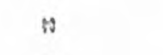 & & Lono Ster Goysor. Yollowatono National Park. \\
\hline 664 & & $"$ & & Old Folthiul Gegsor. Iollowstone National Park \\
\hline
\end{tabular}




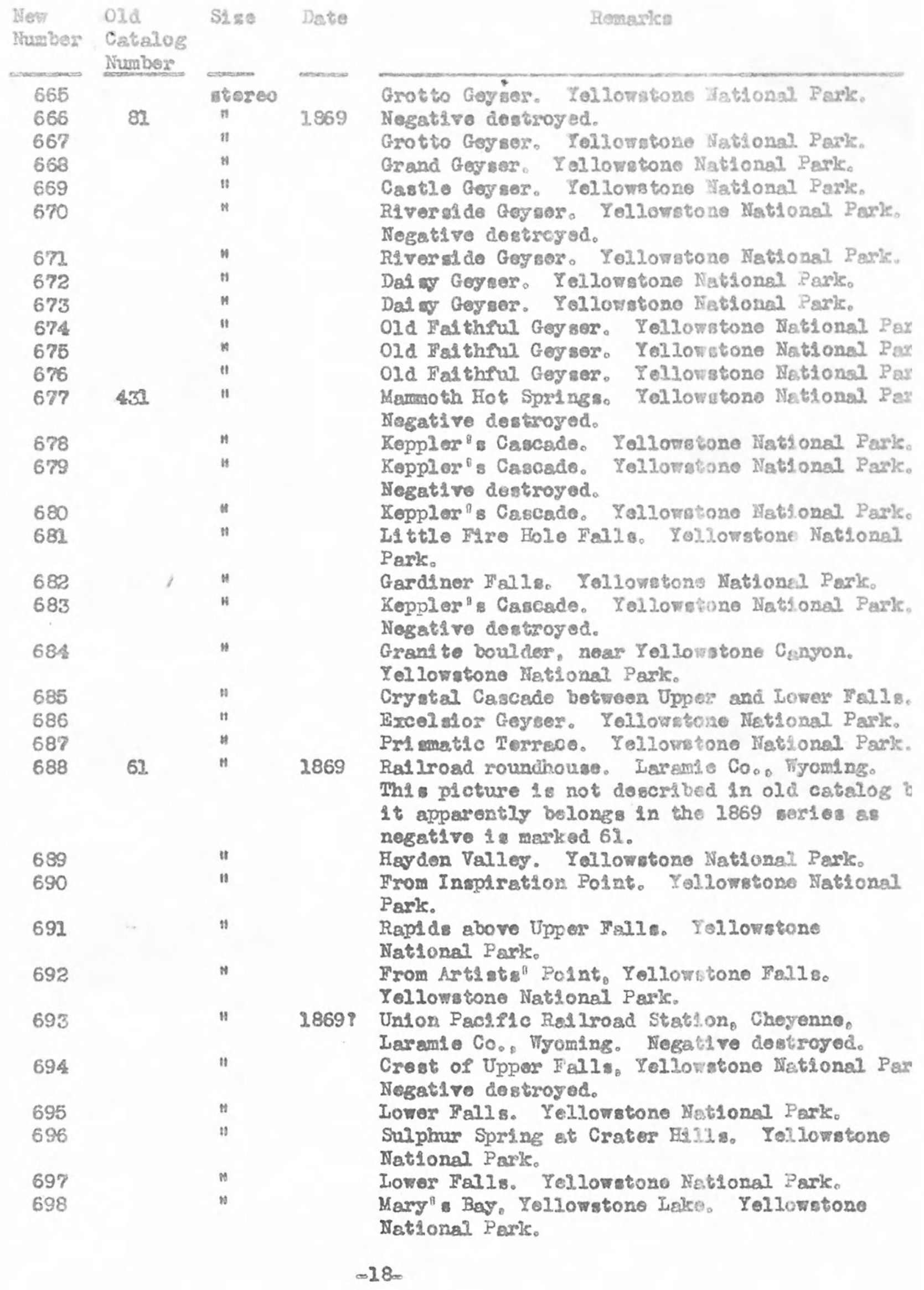




\begin{tabular}{|c|c|c|c|c|}
\hline $\begin{array}{l}\text { Nov' } \\
\text { Number }\end{array}$ & $\begin{array}{l}\text { Old } \\
\text { Catalog } \\
\text { Number. }\end{array}$ & Size & Date & Remexka \\
\hline 699 & & stereo & & $\begin{array}{l}\text { Southest shore, Yollowstone I.ale. Yollowstone } \\
\text { National Park. }\end{array}$ \\
\hline 700 & & n & & $\begin{array}{l}\text { Groat Fountatu Geyser. Tellovstone National } \\
\text { Park. }\end{array}$ \\
\hline 701 & & n & & $\begin{array}{l}\text { O1d Rasthinl Gayser. Yolzongtone Net1onal Park. } \\
\text { Hegative destroyed. }\end{array}$ \\
\hline 202 & & " & & $\begin{array}{l}\text { Bis Blue Springo Lower Basin. Yollowstone } \\
\text { Hational Pari. Negastve destroyed. }\end{array}$ \\
\hline 703 & & " & & Iower Barin. Yollowatone Nat̂lonsl Parik. \\
\hline 204 & 60 & " & 1869 & \\
\hline 705 & & म & & Lower Bastn. Yellowstome Natlonal Park. \\
\hline 706 & $503 ?$ & $n$ & 2872 & \\
\hline 707 & & $n$ & & $\begin{array}{l}\text { Coatsing speciacrs. Mammoth Hot Springs. } \\
\text { Yollowntone National Park. }\end{array}$ \\
\hline 707 & & $5 x^{7} 7$ & & $\begin{array}{l}\text { Seme caption as ro7. This piçure is of a } \\
5 x 7 \text { ondargement. }\end{array}$ \\
\hline 708 & 62 & stez:o & 1869 & 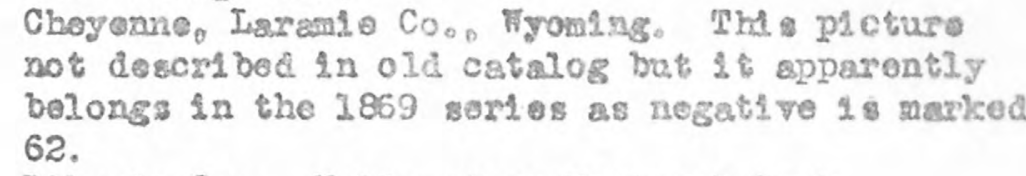 \\
\hline 709 & & n & & Ifborty Cay. Yellowstone National Park. \\
\hline 710 & & " & & $\begin{array}{l}\text { Pulps Torrace, Hawnoth Hot Springas. } \\
\text { Yol. }\end{array}$ \\
\hline 71 & & $n$ & & $\begin{array}{l}\text { Photo outelt at gurimit of } M t \text {. Fashburn. } \\
\text { Peliowstone National Park. }\end{array}$ \\
\hline 722 & 65 & $\ddot{n}$ & 1859 & Pulp1: Texraces, Mamoth Hot Spz \\
\hline 713 & & $\mathrm{~m}$ & & Yellowstone Nat1onal Park. \\
\hline $\begin{array}{l}714 \\
75\end{array}$ & 66 & " & 2869 & Mamoth Fot Springm。 Yollowetone National Park. \\
\hline 76 & & $5 \times 7$ & 1869 & $\begin{array}{l}\text { A } 5: 7 \text { onlargenent of now numbor } 776 \text { (old catalog } \\
\text { maber } 68 \text { ). }\end{array}$ \\
\hline 727 & & storeo & & Mamoth Hot Springs. Tal Iowetone Natsonal Park. \\
\hline 778 & 70 & " & 1869 & \\
\hline 719 & & $"$ & & $\begin{array}{l}\text { Crosalng Bear RIver. Salt Lake Vallog, Boxelder } \\
\text { County. Utsh. Hegative destroyed. }\end{array}$ \\
\hline 720 & $71 ?$ & $"$ & 2869 & \\
\hline 721 & $71 ?$ & $H$ & $n$ & NegatIve destroyed. \\
\hline 722 & $72 ?$ & $"$ & $"$ & Nesative destroyod. \\
\hline 723 & & $"$ & & Iower Iellowstone. Yellowntone National Park. \\
\hline 724 & & " & & $\begin{array}{l}\text { Lower Yollowatone Falls, Yollowatone National } \\
\text { Park. Negative destrojed. }\end{array}$ \\
\hline 725 & & " & & $\begin{array}{l}\text { Artlsts Point. Yollowstono Caryon. Yellowstone } \\
\text { Ratlonal Park. }\end{array}$ \\
\hline 726 & & " & & Uppor IIalla Yellowstono Natlonsl Park。 \\
\hline 727 & & $"$ & 1859 & $\begin{array}{l}\text { This picture is a near duplicate of nem number } \\
728 \text { (old catalos mumber } 77 \text { of } 1869 \text { serles). }\end{array}$ \\
\hline 728 & & " & & $\begin{array}{l}\text { From brink of Iower Falls. Yellowstone } \\
\text { Wetional Park. }\end{array}$ \\
\hline
\end{tabular}




\begin{tabular}{|c|c|c|c|c|}
\hline $\begin{array}{l}\text { Nen } \\
\text { Number }\end{array}$ & $\begin{array}{l}\text { Ojd } \\
\text { Catarog } \\
\text { Nambor }\end{array}$ & S120 & Desto & Remarkite \\
\hline 729 & 77 & 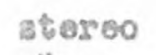 & 1869 & \\
\hline 730 & & & & $\begin{array}{l}\text { This ploture is similar to new muber } 731 \text { (old } \\
\text { catalos number } 78 \text { of } 1869 \text { sertes). }\end{array}$ \\
\hline 732 & 78 & $n$ & s & Nogative destroyed. \\
\hline 732 & & " & & $\begin{array}{l}\text { Castle Geyser, Yellowstone Natzonel Park. } \\
\text { Negative destroyed. }\end{array}$ \\
\hline 733 & & n & " & 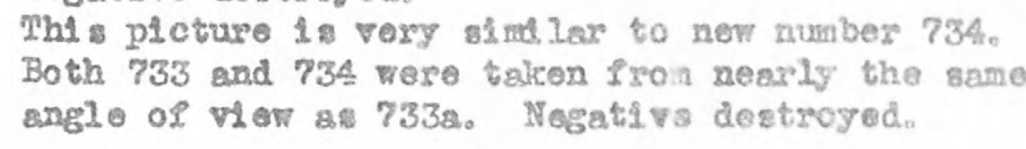 \\
\hline 7338 & 79 & $n$ & n & \\
\hline 734 & & n & " & See remarks 10110w1 ng 733. \\
\hline 735 & 80 & $"$ & 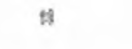 & \\
\hline 736 & & " & & Glant Gyyer. Fellowstone NEtional Park. \\
\hline 737 & 81. & " & $"$ & \\
\hline 738 & & " & & $\begin{array}{l}\text { Geyser. Tellowstone Natlonal Paxic. Nogativo } \\
\text { destroyod. }\end{array}$ \\
\hline 739 & & n & 9369 & $\begin{array}{l}\text { Th1 s plcture 18 almllar to nev mumber } 737 \text { (old } \\
\text { catalog number } 81 \text { of } 1869 \text { mextes). }\end{array}$ \\
\hline 740 & 82 & $"$ & " & Negative destroyed. \\
\hline 7요 & $0 ?$ & " & $n$ & 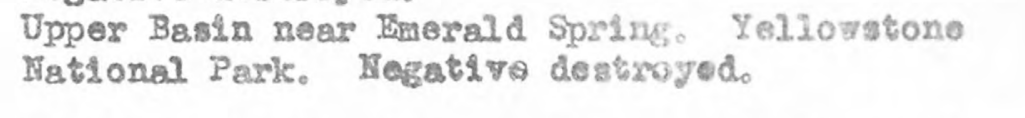 \\
\hline 743 & & " & & $\begin{array}{l}\text { Upper Bastn near Grand. Yollowetone Heslonal } \\
\text { Park. }\end{array}$ \\
\hline 744 & & $n$ & & $\begin{array}{l}\text { Glant Goyser after an exuption. Yellowitone } \\
\text { Nat1onal Park. }\end{array}$ \\
\hline 745 & 85 & s & 2869 & Negative destroged. \\
\hline 746 & & " & " & 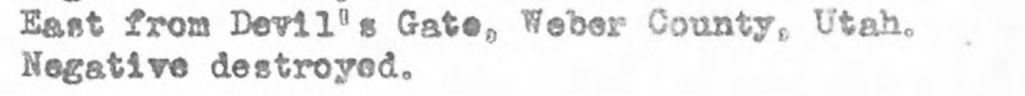 \\
\hline 747 & 86 & $"$ & $"$ & \\
\hline 748 & 87 & is & " & Negatro deskroged. \\
\hline 749 & & n & $" 1$ & $\begin{array}{l}\text { This plcturo is elvilar to new muber } 751 \text { (old } \\
\text { catalog number } 89 \text { of } 1869 \text { sertos). }\end{array}$ \\
\hline 750 & $\begin{array}{l}88 \\
89\end{array}$ & n & " & Negat1ve destrozod. \\
\hline $\begin{array}{l}751 \\
752\end{array}$ & $\begin{array}{l}89 \\
90\end{array}$ & $"$ & ti & Negst1ve dostroyed. \\
\hline $\begin{array}{l}752 \\
753\end{array}$ & 93 & n & $n$ & Nogative deatroyed. \\
\hline 754 & & n & & $\begin{array}{l}\text { Pool of the Excelstor Geymer. Yollowistone } \\
\text { Natlonal Park. Negative deatroyed. }\end{array}$ \\
\hline 756 & 92 & $"$ & $"$ & \\
\hline 756 & 93 & s & $n$ & Nogatlve dentrojed. \\
\hline 757 & 94 & $n$ & " & Negative dowtroyed. \\
\hline 758 & 95 & $n$ & $\omega$ & Nogative destrogod. \\
\hline 759 & 96 & a & $m$ & Negative dontrograd. \\
\hline 760 & 97 & $"$ & $n$ & \\
\hline 761 & 98 & " & " & Negat2vo doasxayod. \\
\hline 762 & 99 & $n$ & $n$ & Nogative dostroyed. \\
\hline 763 & 103 & $n$ & $n$ & \\
\hline
\end{tabular}




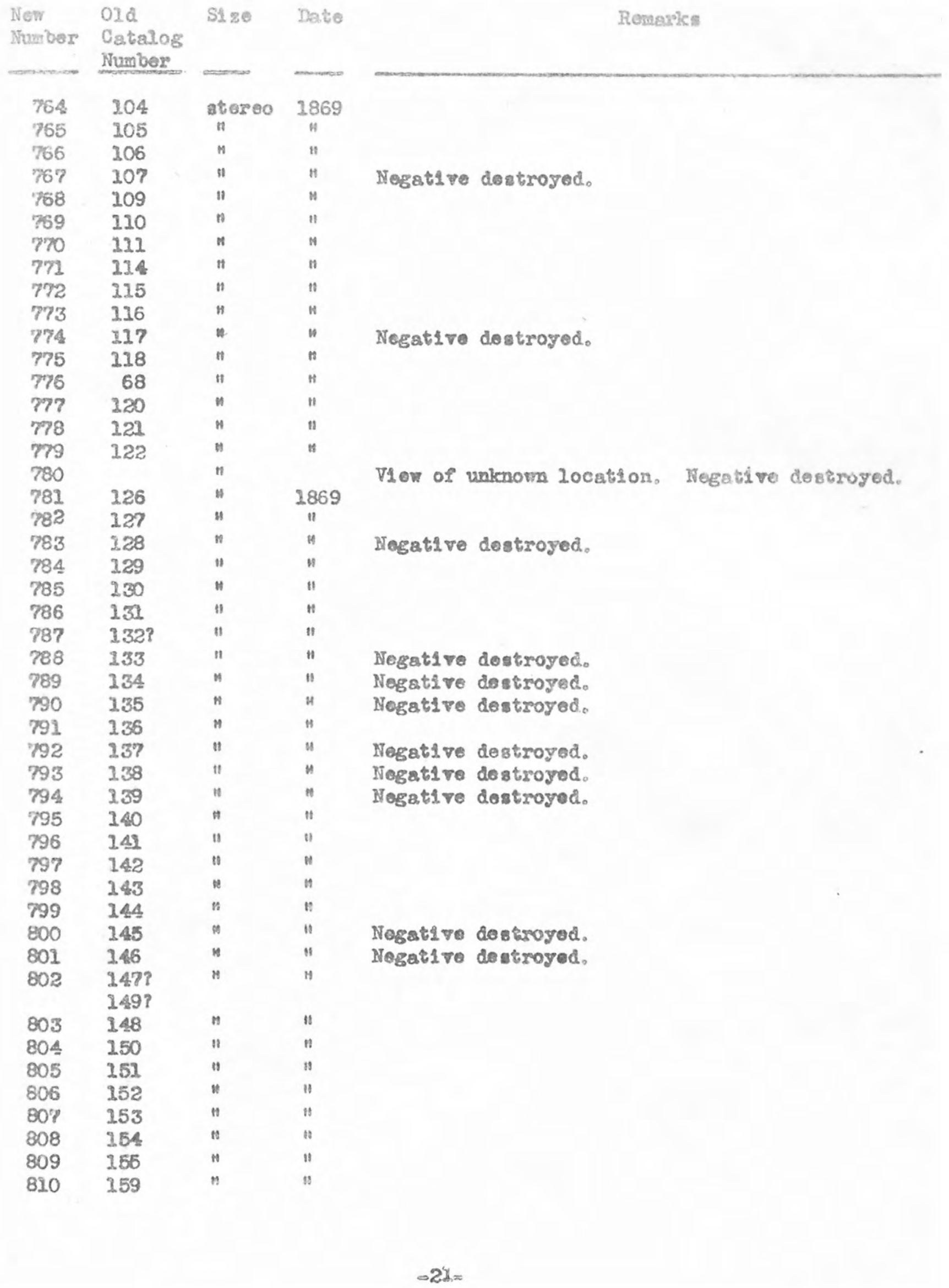




\begin{tabular}{|c|c|c|c|c|}
\hline $\begin{array}{l}\text { Me* } \\
\text { Nusbos }\end{array}$ & $\begin{array}{l}\text { 0ld } \\
\text { Catajog } \\
\text { Number }\end{array}$ & Sizo & Date & Rowarks \\
\hline 81.1 & 160 & stereo & 2869 & \\
\hline 812 & 261 & म & " & \\
\hline 813 & 362 & it & n & \\
\hline 81.4 & $263 ?$ & is & $1869 ?$ & $\begin{array}{l}\text { Caption and subject of this picture ero the ane } \\
\text { ag for new nuber } 117 \% \text {. }\end{array}$ \\
\hline 815 & $364 ?$ & " & 3069 ? & Nogatsto destroyed. \\
\hline 816 & 268 & " & 3869 & \\
\hline 817 & 169 & " & " & \\
\hline 818 & $270 ?$ & " & $2869 ?$ & 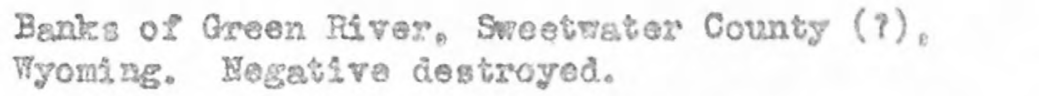 \\
\hline 839 & 2.72 & $n$ & 1869 & \\
\hline 820 & 273 & ต & $H$ & \\
\hline 821. & 275 & " & $n$ & \\
\hline 822 & 377 & " & H & \\
\hline 823 & $\$ 78$ & H & $n$ & \\
\hline 828 & 279 & $"$ & u & Negst1ve destroged. \\
\hline 825 & 180 & $"$ & $n$ & \\
\hline 826 & 181 & $"$ & $n$ & \\
\hline 827 & 182 & $"$ & n & Negat1ve destroyeå. \\
\hline 828 & 283 & $n$ & $"$ & \\
\hline 829 & & " & $"$ & $\begin{array}{l}\text { Shorman Statsion Roundhouse, AI bany Countz. } \\
\text { Fyon? rago Negatve destroyed. }\end{array}$ \\
\hline 830 & 184 & $"$ & $"$ & \\
\hline 832. & & n & a & $\begin{array}{l}\text { Thls plobure stmilas to new muber } 832 \text { (old } \\
\text { catalog maber } 185 \text { of } 1869 \text { sextas). }\end{array}$ \\
\hline 832 & 285 & " & " & \\
\hline 833 & 187 & $n$ & u & Nigative destroyed. \\
\hline 834 & 188 & $n$ & $n$ & \\
\hline 835 & 392 & $"$ & $n$ & Negative destroyed. \\
\hline 836 & 193 & * & $u$ & \\
\hline 837 & 294 & $"$ & 4 & \\
\hline 838 & 195 & $n$ & $n$ & Negestre destroyed. \\
\hline 839 & 296 & " & $"$ & Negative deztroyed. \\
\hline 840 & 197 & " & $"$ & Nagative destrojed. \\
\hline 829 & 198 & " & " & \\
\hline 842 & 199 & $"$ & $n$ & Nogstive destroyed. \\
\hline 843 & 250 & 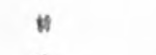 & * & \\
\hline 844 & 252 & $n$ & $"$ & \\
\hline 845 & 253 & $"$ & $"$ & Negat:re dostroyod. \\
\hline 846 & 254 & $"$ & $n$ & \\
\hline 847 & 255 & $n$ & H & Negative destroyed. \\
\hline 848 & 256 & $"$ & H & Negatsvo destroyed. \\
\hline 849 & 257 & $"$ & " & \\
\hline 850 & 259 & $"$ & $n$ & \\
\hline 851 & 260 & " & w & \\
\hline 852 & 261 & H & $\|$ & \\
\hline 853 & 282 & " & $"$ & Nogarive mîsing. \\
\hline 854 & 263 & 1. & $"$ & \\
\hline
\end{tabular}




\begin{tabular}{|c|c|c|c|c|}
\hline $\begin{array}{l}\text { Now } \\
\text { Nowbers }\end{array}$ & $\begin{array}{l}\text { Old } \\
\text { Catalog } \\
\text { Nusbors }\end{array}$ & 5180 & $D_{\text {ate }}$ & Rearaxks \\
\hline 855 & $26 \mathrm{~A}$ & storeo & 1869 & \\
\hline 856 & 272 & $"$ & 1870 & \\
\hline 857 & & $H$ & & $\begin{array}{l}\text { Bot Spring Cone in Yellowstone Lake near Thumb. } \\
\text { Yollovatone Natlonal Perk. }\end{array}$ \\
\hline 858 & 275 & $n$ & 1870 & \\
\hline 859 & 282 & $"$ & " & \\
\hline 860 & 283 & $"$ & $n$ & \\
\hline 861 & 286 & $n$ & " & \\
\hline 862 & 289 & * & $"$ & \\
\hline 863 & 295 & $n$ & n & \\
\hline 864 & 296 & $n$ & $n$ & \\
\hline 865 & 124 & n & 2869 & \\
\hline 866 & 112 & " & $"$ & \\
\hline 867 & & & & Number 867 is unassignod。 \\
\hline 868 & & $"$ & & Hesd of Buakskin Guiloh. Paxk Cotnty, Colorado. \\
\hline 869 & 302 & $"$ & 1870 & Negative destroyed. \\
\hline 870 & 306 & $" 1$ & N & \\
\hline $8 n$ & 307 & w & n & \\
\hline 872 & 308 & " & " & \\
\hline 873 & 316 & " & n & \\
\hline 874 & 327 & n & n & \\
\hline 875 & 351 & $"$ & $n$ & \\
\hline 876 & 353 & * & n & \\
\hline 877 & 358 & $n$ & $n$ & \\
\hline 878 & 330 & 11 & " & \\
\hline 879 & 339 & " & " & \\
\hline 880 & 350 & $n$ & $n$ & \\
\hline 881 & 325 & " & " & \\
\hline 882 & 352 & n & $"$ & \\
\hline 883 & 354 & ต & " & \\
\hline 884 & & $n$ & " & 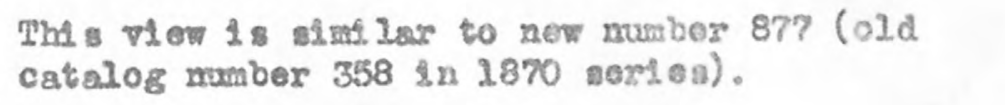 \\
\hline 886 & 359 & n & n & \\
\hline 886 & & " & " & Kounts ${ }^{0}$. Douglas County. Colorado. \\
\hline 887 & 360 & $"$ & s & \\
\hline 888 & 361 & " & $n$ & \\
\hline 889 & 363 & $n$ & $\begin{array}{l}n \\
n\end{array}$ & \\
\hline 890 & 364 & n & $\begin{array}{l}n \\
\text { " }\end{array}$ & \\
\hline 891 & 366 & n & " & \\
\hline 892 & 367 & n & $\begin{array}{l}n \\
n\end{array}$ & \\
\hline 893 & 369 & $\omega$ & " & \\
\hline 894 & 370 & n & $\begin{array}{l}n \\
n\end{array}$ & \\
\hline 895 & 373 & n & $\begin{array}{c}1 \\
1870 ?\end{array}$ & \\
\hline 896 & $379 ?$ & n & $1870 \%$ & $\begin{array}{l}\text { Stevenson, Turnbull, Elliott, Braneon, Potato } \\
\text { John, (@ooks. }\end{array}$ \\
\hline 897 & 381 & n & 1871 & \\
\hline 898 & 388 & $n$ & $"$ & Negative destroged. \\
\hline 899 & 390 & $\omega$ & $n$ & Nogative destroyod. \\
\hline
\end{tabular}




\begin{tabular}{|c|c|c|c|c|}
\hline $\begin{array}{l}\text { Ker } \\
\text { Nusuber }\end{array}$ & $\begin{array}{l}\text { Old } \\
\text { Cataiog } \\
\text { Numbor }\end{array}$ & S2ze & Dato & Rensreles \\
\hline 900 & 391 & ஐเละชอ & 1871 & Negat1va dostroyed. \\
\hline 901 & 392 & N & H & Negative destroged. \\
\hline 902 & 394 & $n$ & $n$ & Nogat1ve dostroyad. \\
\hline 903 & 395 & $n$ & 11 & \\
\hline 904 & 397 & $"$ & * & \\
\hline 905 & 399 & n & $n$ & \\
\hline 906 & 400 & H & " & \\
\hline 907 & 401 & $"$ & * & \\
\hline 908 & 402 & $"$ & H & \\
\hline 909 & 103 & $n$ & $" 1$ & \\
\hline 910 & 404 & $n$ & n & \\
\hline 911 & 405 & $n$ & $n$ & \\
\hline 912 & 406 & $"$ & " & \\
\hline 913 & 407 & * & и & \\
\hline 914 & & " & & 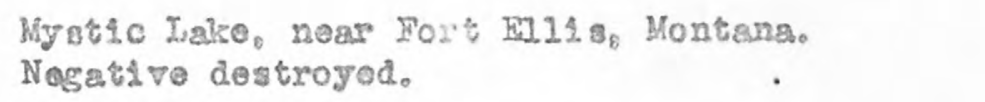 \\
\hline 915 & 410 & $"$ & 1872 & \\
\hline 926 & 411 & $n$ & * & \\
\hline 917 & 473 & " & " & \\
\hline 918 & 414 & $u$ & $"$ & \\
\hline 919 & 419 & n & 月 & \\
\hline 920 & $\triangle 20$ & $"$ & n & \\
\hline 921 & 424 & u & " & \\
\hline 922 & 427 & * & "1 & \\
\hline 923 & 428 & $"$ & $n$ & \\
\hline 924 & $4: 36$ & H & $"$ & $\begin{array}{l}\text { Cap of Liberty of Gardiner" in River. Hamoth } \\
\text { Hot Sprtaga Xellowistone Nathonal Park. } \\
\text { Nogative destroyed. }\end{array}$ \\
\hline 925 & 438 & $"$ & n & \\
\hline 926 & & n & & $\begin{array}{l}\text { Black Sand Pool. Yellowntone Natlonal Park. } \\
\text { Nogat1vo destrojed. }\end{array}$ \\
\hline 927 & $\Delta 56$ & $" 1$ & 9871 & \\
\hline 928 & 484 & $n$ & $" 1$ & \\
\hline 929 & 482 & $n$ & $" 1$ & Negst1to destroyed. \\
\hline 930 & & $n$ & 1872 & $\begin{array}{l}\text { Thi a plcture is a moar duplicato of new maber } \\
931 \text { (old catalog muber } 494 \text { of } 1872 \text { versos). } \\
\text { Nogative dostrogod. }\end{array}$ \\
\hline 932. & 494 & $n$ & (6) & \\
\hline 932 & 495 & n & $"$ & \\
\hline 933 & 502 & H & $n$ & \\
\hline 934 & 504 & $"$ & " & \\
\hline 935 & & $"$ & $"$ & $\begin{array}{l}\text { This piotuse is a near duplicate of new muber } \\
934 \text { (old catalog numbr } 504 \text { of } 1872 \text { gerles). } \\
\text { Negative destroged. }\end{array}$ \\
\hline 936 & 506 & $" 1$ & $"$ & \\
\hline 937 & 510 & $n$ & " & \\
\hline 938 & 511 & " & $n$ & Negative destroyed. \\
\hline 939 & 514 & $H$ & $"$ & \\
\hline
\end{tabular}




\begin{tabular}{|c|c|c|c|c|}
\hline $\begin{array}{l}\text { Now } \\
\text { Nuaber }\end{array}$ & $\begin{array}{l}\text { Old } \\
\text { Catalog } \\
\text { Nurnogr }\end{array}$ & S120 & Dato & Reanska \\
\hline 940 & & gtoreo & 1872 & $\begin{array}{l}\text { Thi a plcture is a nees duplicate of new number } \\
939 \text { (old catalog muber } 514 \text { of } 1872 \text { series). } \\
\text { Wegative destroyed. }\end{array}$ \\
\hline 941 & 515 & $"$ & " & \\
\hline 942 & 518 & $n$ & $n$ & \\
\hline 943 & 519 & $H$ & $"$ & Negative destroyed. \\
\hline 944 & & $"$ & $n$ & $\begin{array}{l}\text { This plcture is a nesx duplicate of new number } \\
943 \text { (old catalog number } 519 \text { of } 1872 \text { sorteg). } \\
\text { Nogst1ve destroyed. }\end{array}$ \\
\hline 945 & 52ณ & $n$ & $n$ & \\
\hline 946 & & " & $"$ & 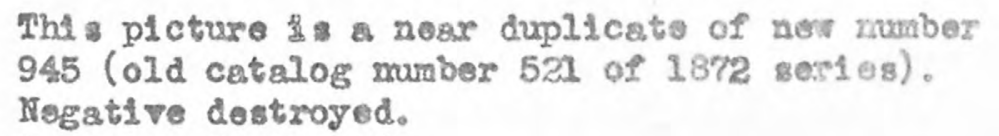 \\
\hline 947 & $524 ?$ & $n$ & $2872 ?$ & Negat1ve destroyed. \\
\hline 948 & 525 & $n$ & 1872 & \\
\hline 949 & 528 & $"$ & n & \\
\hline 950 & 533 & " & " & \\
\hline 951 & $534 ?$ & $"$ & " & Megative dostroyed. \\
\hline 952 & & & & Kumber 95218 une \\
\hline 953 & 536 & " & " & \\
\hline 954 & & $" 1$ & & $\begin{array}{l}\text { Pool in Lower Basin. Yallowtone National Park. } \\
\text { Negat1ve destroyed. }\end{array}$ \\
\hline 955 & 541 & " & $\$ 872$ & Negative destroyed. \\
\hline 956 & 550 & * & n & \\
\hline 957 & 551 & " & H & \\
\hline 958 & 554 & * & " & \\
\hline 959 & 545 & $n$ & " & Negative degtroyed. \\
\hline 960 & 569 & n & $"$ & \\
\hline 961 & 573 & n & $"$ & Negative destroged. \\
\hline 962 & 578 & $M$ & $n$ & \\
\hline 963 & 581 & " & " & \\
\hline 964 & $585-$ & $"$ & $n$ & \\
\hline 965 & & & & Nrumber 965 I: uns.stened. \\
\hline 966 & 587 & " & $n$ & Negative destroyed. \\
\hline 967 & 586 & n & " & \\
\hline 968 & 558 & n & s & \\
\hline 969 & 590 & $"$ & n & \\
\hline 970 & 591 & $"$ & $"$ & \\
\hline 972 & 563 & n & $"$ & Nogative destrogad. \\
\hline 972 & 592 & $"$ & $n$ & Negative destroyad. \\
\hline 973 & 593 & $n$ & $"$ & \\
\hline 974 & 596 & $"$ & n & Nogative destroyed. \\
\hline 975 & 597 & n & $n$ & \\
\hline 976 & 598 & $n$ & n & Negative destroyed. \\
\hline 977 & 599 & $n$ & " & \\
\hline 978 & 258 & $" 1$ & 1869 & $\begin{array}{l}\text { View above the hoad of Crow Craek. Iaramis } \\
\text { County. Wyoming. }\end{array}$ \\
\hline 979 & 600 & $"$ & 1872 & \\
\hline
\end{tabular}




\begin{tabular}{|c|c|c|c|c|}
\hline $\begin{array}{l}\text { Hew } \\
\text { Numbor }\end{array}$ & $\begin{array}{l}\text { Catalog } \\
\text { Number }\end{array}$ & Sil zo & De.te & Remarics \\
\hline 980 & 601 & storeo & 3872 & \\
\hline 981 & & (3) & H & $\begin{array}{l}\text { This plcture is a near duplicete of new muber } \\
980 \text { (old catalog mabor } 601 \text { of } 1872 \text { sorsos). }\end{array}$ \\
\hline 982 & 603 & H & "11 & Negaisve destroyed. \\
\hline 983 & & n & " & $\begin{array}{l}\text { This pictura is a nesx dupl1 cate of new rumber } \\
984 \text { (old catalog munber } 604 \text { of } 1872 \text { aerles). }\end{array}$ \\
\hline 984 & 604 & $n$ & " & \\
\hline 985 & 605 & " & $"$ & \\
\hline 986 & 606 & H & $"$ & \\
\hline 987 & 609 & $"$ & " & \\
\hline 988 & 63.0 & $"$ & n & \\
\hline 989 & & u & 4 & $\begin{array}{l}\text { This plcture ta s near dupl1 csto of now nunber } \\
1035 \text { (old catalog mubor } 611 \text { of } 1872 \text { sertog). }\end{array}$ \\
\hline 990 & 637 & $"$ & $"$ & \\
\hline 991 & 618 & $n$ & $"$ & \\
\hline 992 & 622 & 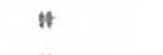 & $"$ & NogacL vo mis B Ine \\
\hline 993 & 623 & $"$ & $"$ & \\
\hline 994 & 624 & $"$ & " & \\
\hline 995 & 625 & $"$ & " & \\
\hline 996 & 632 & $"$ & N & \\
\hline 997 & 933 & $"$ & 1875 & \\
\hline 998 & 641 & a & 2873 & \\
\hline 999 & 645 & $"$ & $"$ & \\
\hline 1000 & 647 & $"$ & $n$ & \\
\hline 2003 & 649 & " & $"$ & \\
\hline 1002 & 650 & H & $H$ & \\
\hline 1003 & 653 & $" 1$ & $n$ & \\
\hline 1004 & 660 & " & $"$ & \\
\hline 1.005 & 666 & " & n & Nogattro destrojod. \\
\hline 1006 & 667 & $"$ & " & Negative destroyed. \\
\hline 1.007 & 672 & $"$ & " & \\
\hline 1008 & 673 & $n$ & H & \\
\hline 1009 & 675 & $"$ & $n$ & \\
\hline 1010 & 678 & $*$ & $"$ & \\
\hline 1012 & 679 & " & $"$ & Negat1ve destroyed. \\
\hline 1012 & 680 & $" 1$ & $"$ & Nagative dostroyed. \\
\hline 1013 & 682 & $"$ & $n$ & \\
\hline $10 \% 4$ & & 18 & H & $\begin{array}{l}\text { Sinilar to new muber } 1022 \text { (old catalog maber } \\
690 \text { ). Nogativo destroyed. }\end{array}$ \\
\hline 115 & 683 & $n$ & $"$ & \\
\hline 2016 & 684 & 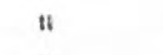 & $H$ & \\
\hline 1017 & 686 & $"$ & $"$ & Those are ortghnal duplicate negetives. \\
\hline 2018 & 686 & $"$ & $"$ & Thoso are oxiglnal duplicato nogatives. \\
\hline 2019 & 687 & " & $"$ & \\
\hline 2020 & 688 & " & " & \\
\hline 1021 & 689 & $"$ & $"$ & Negative destroyed. \\
\hline 1022 & 690 & $n$ & " & Nogat1vo destroged. \\
\hline 1023 & 692 & $"$ & $n$ & \\
\hline 1024 & 695 & h & $"$ & \\
\hline
\end{tabular}




\begin{tabular}{|c|c|c|c|}
\hline $\begin{array}{l}\text { New } \\
\text { Nowbex }\end{array}$ & $\begin{array}{l}\text { OId } \\
\text { Catalos } \\
\text { Rembeo }\end{array}$ & \$2 20 & Deto \\
\hline 2025 & 696 & sto200 & 2873 \\
\hline 1026 & 697 & $n$ & \\
\hline 2027 & 697 & " & $n$ \\
\hline 1028 & 698 & $"$ & $n$ \\
\hline 1029 & 699 & $M$ & n \\
\hline 1030 & 200 & " & " \\
\hline 1031 & 700 & $"$ & n \\
\hline 2032 & 702 & H & $" 1$ \\
\hline 2033 & Tos & H & ต \\
\hline 1034 & 704 & $n$ & " \\
\hline 1035 & 611 & n & 1872 \\
\hline 1036 & 706 & $n$ & 1873 \\
\hline 1037 & & $n$ & \\
\hline 1038 & 707 & " & $n$ \\
\hline 1039 & 708 & $M$ & и \\
\hline 1040 & n10 & $n$ & $n$ \\
\hline 1041 & no & $n$ & $n$ \\
\hline 1042 & 71 & $n$ & $n$ \\
\hline 2043 & 71 & n & $H$ \\
\hline 2044 & n4 & $"$ & $"$ \\
\hline 1045 & 715 & " & $" 1$ \\
\hline 1046 & 717 & $n$ & " \\
\hline 1047 & 720 & " & 1874 \\
\hline $20 A 8$ & 721 & $n$ & " \\
\hline 1049 & 727 & $n$ & " \\
\hline 1050 & 723 & $n$ & $n$ \\
\hline 2051 & 724 & $n$ & $n$ \\
\hline 2052 & 725 & $n$ & $n$ \\
\hline 1053 & 726 & " & $H$ \\
\hline 2054 & & $n$ & $n$ \\
\hline 2055 & 728 & n & n \\
\hline 1056 & 729 & * & $n$ \\
\hline 1057 & 730 & H & $n$ \\
\hline 1058 & 731 & H & $n$ \\
\hline 1059 & 732 & n & $H$ \\
\hline 1060 & 733 & " & $n$ \\
\hline 1061 & 734 & N & $n$ \\
\hline 1062 & $735 ?$ & " & H \\
\hline 2063 & 7358 & " & H \\
\hline 1064 & 737 & $n$ & ต \\
\hline 1065 & 738 & " & $n$ \\
\hline 1066 & 739 & $n$ & $n$ \\
\hline 1067 & $7 A O$ & N & ต \\
\hline 1068 & $7 A 1$ & is & H \\
\hline
\end{tabular}

These are origtnal duplicate nogañvo

Thoso aro oxiginsi duplicsto negatives.

These are orf glnal duplicate nogatives.

These are ortglnal duplloate negatives.

Jiegative destroyed.

This pioture is a noax dupl1 cate of nev number 1038 (old cetalos numbr 7or of 1873 geries).

Those are original dupllcato nogatives。 The are original duplicste negstives. These are ortglnal duplicate nogatives. Those aro orlglnal duplicate nogatlvos.

This plcture is a noar duplicate of new muber 1049 (old catalog number rat? of 1874 series).

Negativo destroged.

Megative deatroyed.

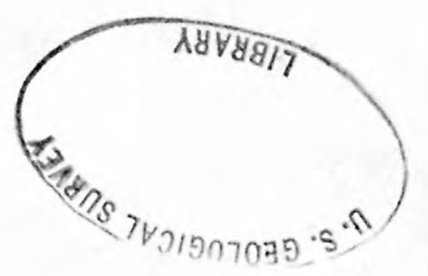

Plctures mubered 1067 and 2167 are printa from original duplicato negatives and ench negative Is maxked with old catalog number 790 .

Plctures numbered 1068 and 2.168 are prints from original duplicate negatives and each nogative 2. maxked wh old satalos muber 741 . 


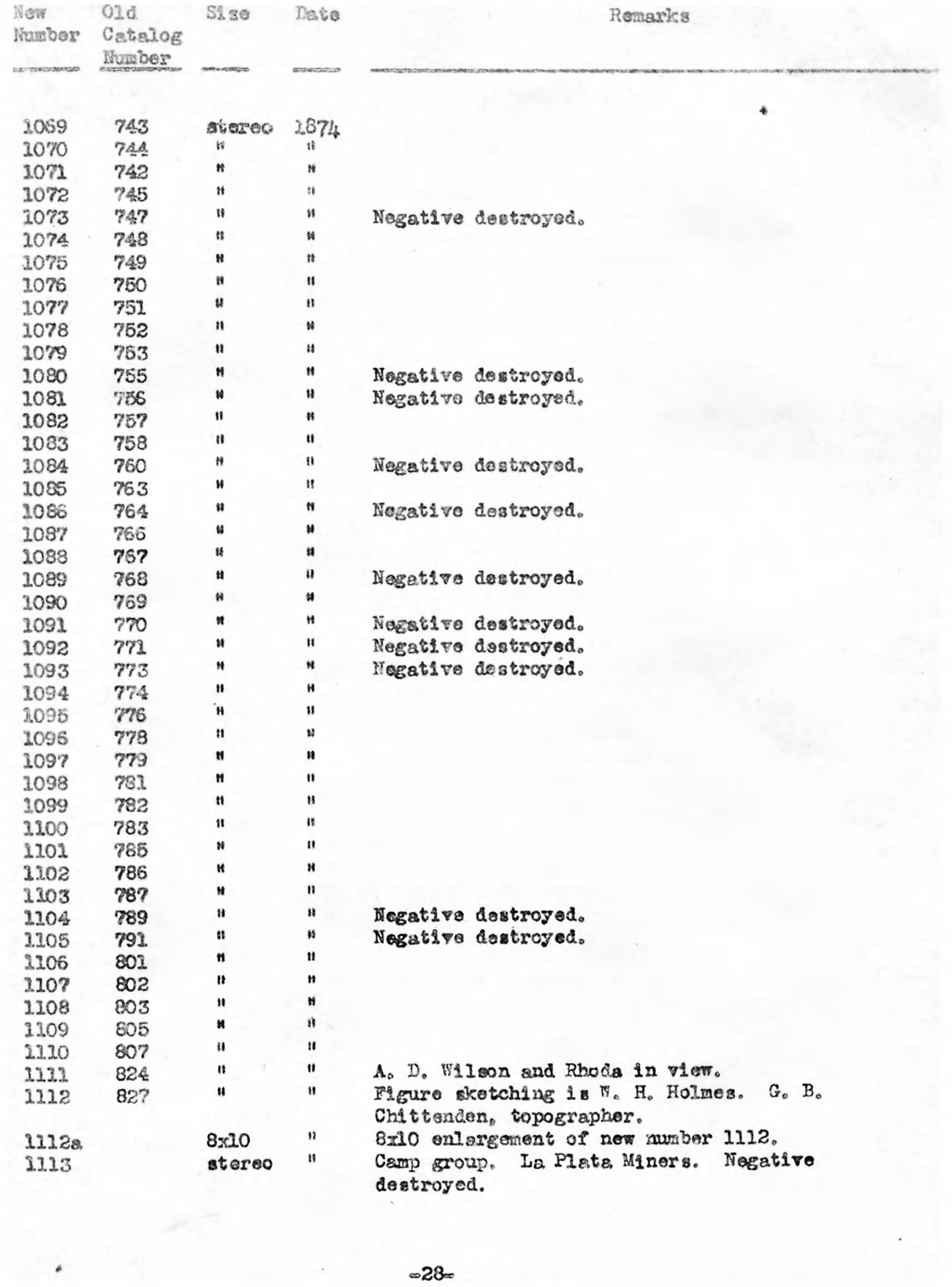




\begin{tabular}{|c|c|c|c|c|}
\hline $\begin{array}{l}\text { New } \\
\text { Ninn ber }\end{array}$ & $\begin{array}{l}\text { Old } \\
\text { Cataiog } \\
\text { Thuaber }\end{array}$ & S1:ge & Daţo & Remarka \\
\hline 1214 & & stereo & 1878 & $\begin{array}{l}\text { Camp group. Captairs Jobn Mosi. Negative } \\
\text { do wrogod. }\end{array}$ \\
\hline 2115 & 863 & $"$ & n & La Plata miners ${ }^{\theta}$ compo \\
\hline 3216 & 865 & " & $"$ & Mro $W_{0} \mathrm{~B}_{0}$ Jackson on pony. \\
\hline $311 \%$ & & $n$ & & $\begin{array}{l}\text { Comp scone. (Tht scene shows a sroup of pock } \\
\text { antual is.) Negative destroyod. }\end{array}$ \\
\hline 2118 & & " & & $\begin{array}{l}\text { Camp scone. Scone ahoms a whito horse and } \\
\text { Iligurs of unidentifled man. Nogative dagtroyed. }\end{array}$ \\
\hline 1119 & & " & & $\begin{array}{l}\text { Camp scene. Figure is Dr. Haydon. Ilegative } \\
\text { destroged. }\end{array}$ \\
\hline 1.20 & & * & & $\begin{array}{l}\text { Cam scens. Higure is Jas. Stromsono Nagative } \\
\text { de troged. }\end{array}$ \\
\hline 1121 & & " & & $\begin{array}{l}\text { Camp sceno. Figure is Dr. Turnball. Nagative } \\
\text { destroyed. }\end{array}$ \\
\hline 1122 & & " & 1870 ? & $\begin{array}{l}\text { Ford in view. This man was only on the } 18 \% 0 \\
\text { expedition. }\end{array}$ \\
\hline 3123 & & " & & $\begin{array}{l}\text { Camp scene. Hgure is Carrington. Hogative } \\
\text { destroged. }\end{array}$ \\
\hline 1124 & & n & & $\begin{array}{l}\text { Comp scene. Figure is Schnt t̂. Nogetive } \\
\text { dostroged. }\end{array}$ \\
\hline 1125 & & " & 18709 & $\begin{array}{l}\text { Higure if } S_{0} R_{0} \text { Glfford the artist. This mans } \\
\text { was only on the } 1870 \text { expodition. }\end{array}$ \\
\hline 1126 & & " & & 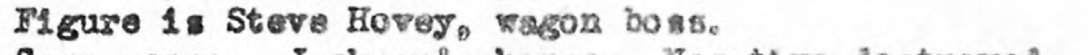 \\
\hline 1127 & & $n$ & & Camp scone, Jackson" bors: Wegative destroyed. \\
\hline 2128 & & $"$ & & Camp scene. Trumball ${ }^{\mathrm{g}} \mathrm{s}$ hosse. Negative destrogad. \\
\hline 1129 & & " & $1870 ?$ & $\begin{array}{l}\text { Camp Stambaugh (Sorath Pass 1870). Negetivs } \\
\text { dostroyed. }\end{array}$ \\
\hline 1.130 & & $"$ & & 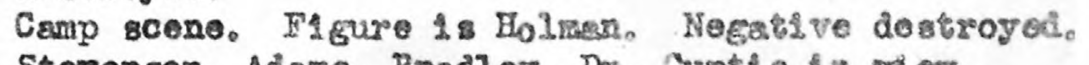 \\
\hline 1131 & & " & $1872 ?$ & 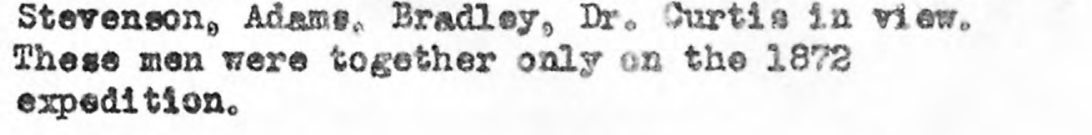 \\
\hline 2132 & 901 & $"$ & 1875 & \\
\hline 1133 & 902 & H & " & \\
\hline 1134 & 903 & " & 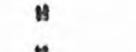 & \\
\hline 1135 & 904 & $n$ & $n$ & Nogat1ve destroyed. \\
\hline 1.136 & 905 & $n$ & $"$ & Negat1 re destroyed. \\
\hline 2137 & 906 & $n$ & $n$ & \\
\hline 2039 & 907 & " & $"$ & \\
\hline $\begin{array}{l}1139 \\
1140\end{array}$ & $\begin{array}{l}911 \\
914\end{array}$ & $n$ & $"$ & Negative deatroyed. \\
\hline 1141 & 925 & $n$ & " & \\
\hline 1142 & 922 & $"$ & $n$ & \\
\hline 11.43 & 923 & 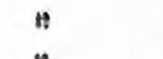 & $n$ & \\
\hline 3144 & $\$ 24$ & " & $n$ & \\
\hline 1145 & 925 & $"$ & $n$ & \\
\hline 1146 & $927 ?$ & $"$ & n & Nagatíve destroyed. \\
\hline 1147 & $931 ?$ & $"$ & $"$ & Hegative destroyed. \\
\hline 1148 & 932 & $n$ & $n$ & \\
\hline
\end{tabular}




\begin{tabular}{|c|c|c|c|c|}
\hline New & $\begin{array}{l}\text { Old } \\
\text { Catalog } \\
\text { Number }\end{array}$ & Size & Deto & Remarks \\
\hline 1149 & $934 ?$ & stereo & 1875 & Negative destroyed. \\
\hline 3350 & 935 & & $"$ & \\
\hline 1151 & 936 & $"$ & $"$ & \\
\hline 1152 & $937 ?$ & $"$ & $"$ & Negative destroyed. \\
\hline 1253 & 940 & " & H & Negative destroyed. \\
\hline 1154 & 942 & " & $"$ & Nogative destroyed. \\
\hline 1155 & 943 & $n$ & " & Negative destroyed. \\
\hline 3156 & 944 & n & H & \\
\hline 1157 & $945 ?$ & " & " & Hegat1vo destroyed. \\
\hline 1158 & 947 & " & " & \\
\hline 1159 & 948 & $"$ & $"$ & \\
\hline 1160 & 953 & " & " & \\
\hline 1161 & 954 & " & " & \\
\hline 1162 & 964 & " & $"$ & E。 A. Barber and Harry Leo in vlow. \\
\hline 1163 & 966 & n & " & \\
\hline 1164 & 97 & " & " & $\begin{array}{l}\text { Fl gure is "Chittonden" a assigtant". Nogativo } \\
\text { destroyed. }\end{array}$ \\
\hline 2165 & 784 & " & 2874 & \\
\hline 1166 & 777 & $"$ & $"$ & \\
\hline 1167 & 740 & " & $"$ & Soe remarks followlng new number $106 \%$ 。 \\
\hline 1268 & 741 & " & " & See renarks following new number 1068。 \\
\hline 1169 & & " & " & $\begin{array}{l}\text { Near duplicate of nev number } 1056 \text { (old catelog } \\
\text { number } 728 \text { of } 1874 \text { serlos). Hegative de stroyod. }\end{array}$ \\
\hline 1370 & & & & Unass? gned. \\
\hline 1171 & & abereo & & $\begin{array}{l}\text { Lake in San Iuf a Valley, Colorado. Nogativo } \\
\text { destroyed. }\end{array}$ \\
\hline 1172 & 662 & $"$ & 2873 & \\
\hline 1173 & & " & & 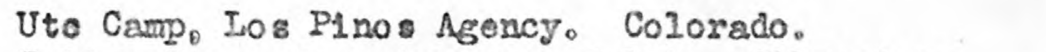 \\
\hline 1174 & & " & 2874 & 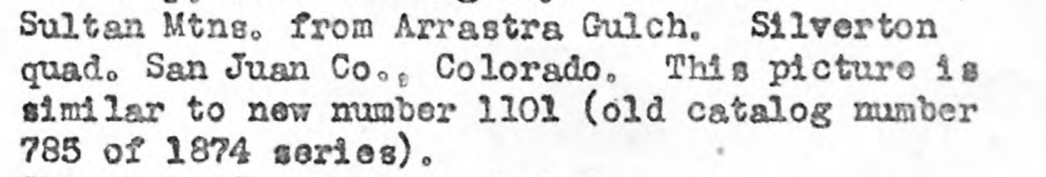 \\
\hline 1375 & & " & & Church in Chshuobus. Mexico. \\
\hline 1176 & & " & & $\begin{array}{l}\text { Preaurably view of MI s sourd (?) River in Hobraska } \\
\text { Negative dentroyed. }\end{array}$ \\
\hline 1177 & $163 ?$ & $"$ & $2869 ?$ & $\begin{array}{l}\text { Caption and subject of this picture are the same } \\
\text { as for new number } 814 \text {. }\end{array}$ \\
\hline 1178 & & $6 \frac{7}{3} \times 8 \frac{1}{2}$ & & 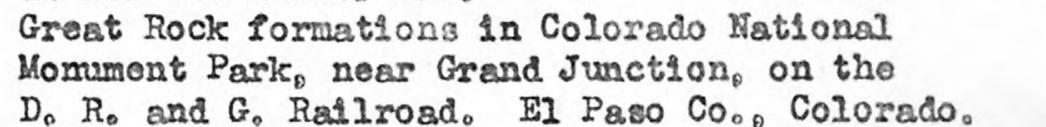 \\
\hline 3179 & & $21 \times 14$ & $2878 ?$ & $\begin{array}{l}\text { Yellowstone Lake, Yellowstone Park. East shore } \\
\text { of Yellowatone Lake showling Promontory Point. }\end{array}$ \\
\hline 1180 & & " & $1878 ?$ & $\begin{array}{l}\text { Yellowstone Lake, Yellowstone Pariz, East shore } \\
\text { of Yollonstone Lake showing the valley of the } \\
\text { Upper Yellowstone River. }\end{array}$ \\
\hline 1381 & & " & $1883 ?$ & $\begin{array}{l}\text { Cratar of Lone Star Geyser, Yellowstons Parke } \\
\text { Arnold Hague on sumult of cone. }\end{array}$ \\
\hline 1182 & 15 & " & 2872 & \\
\hline
\end{tabular}




\begin{tabular}{|c|c|c|c|c|}
\hline $\begin{array}{l}\text { Now } \\
\text { Nrumber }\end{array}$ & $\begin{array}{l}\text { Old } \\
\text { Catalog } \\
\text { Number }\end{array}$ & Sizo & Date & Resarko \\
\hline 2183 & & $11 \times 14$ & $1883 ?$ & Cxater of Glant Geyser. Yallongtone Park. \\
\hline 3284 & & & $1883 ?$ & 01d Fat thrul in act1on. Yellowstone Prok。 \\
\hline $118 s_{a}$ & & $6 \frac{1}{2} 28 \frac{1}{2}$ & $1883 ?$ & Reductilon of new mabor 1184 . \\
\hline 2185 & & 11214 & $1883 ?$ & $\begin{array}{l}\text { Upper Goyzer Basin, Yollowatone Park, from } \\
\text { O1d Fal throl. }\end{array}$ \\
\hline 3.186 & 13 & " & 1872 & \\
\hline 2187 & & H & & Crator of Caetia Geyser. Yellowstone Park. \\
\hline 1288 & & $"$ & $1883 ?$ & $\begin{array}{l}\text { Hot Springs in the Queen }{ }^{n} \text { I Imundry. } \\
\text { Yollowgtone Park. }\end{array}$ \\
\hline 1289 & & & & Tumber 1189 is unse gl gxod. \\
\hline 1190 & 26 & 12xํㅕ욜 & 1872 & \\
\hline 1191 & $28 ?$ & $n$ & $1872 ?$ & \\
\hline 2192 & $24 ?$ & " & $1872 ?$ & \\
\hline 2193 & $27 ?$ & $"$ & $1872 ?$ & \\
\hline 1194 & & $"$ & $1878 ?$ & $\begin{array}{l}\text { Glen Springs, Mamnoth Hot Sprisgs: } \\
\text { Yellowstone Park. }\end{array}$ \\
\hline 2195 & & H & & $\begin{array}{l}\text { Mammoth Hot Spriags, Yallowatone Park, } \\
\text { genoral VIew Irom Fort H121. }\end{array}$ \\
\hline 1796 & & $n$ & 28789 & $\begin{array}{l}\text { Lower portion of Jupltor Ferras, Hammoth Flot } \\
\text { Springs. Iellowstone Fark. Bunon }{ }^{3} \text { Poak in } \\
\text { distance. }\end{array}$ \\
\hline 1197 & & $n$ & 18789 & 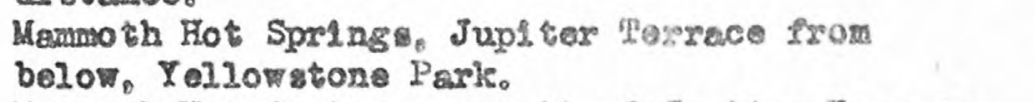 \\
\hline 1198 & & $n$ & $1872 ?$ & 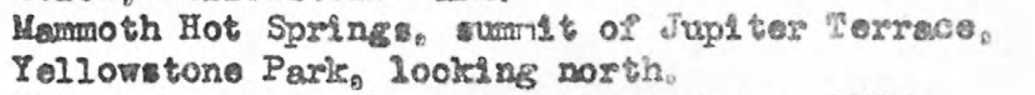 \\
\hline 1199 & & $"$ & $1872 ?$ & 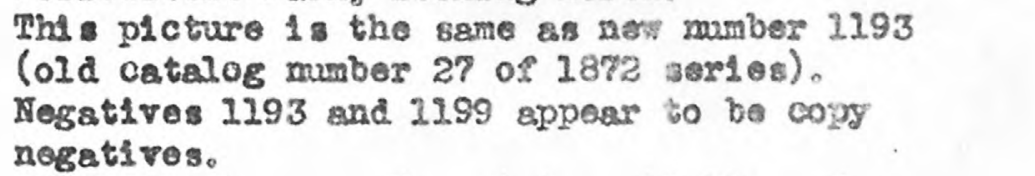 \\
\hline 1200 & & & & $\begin{array}{l}\text { Subjoct unkmown. No print aral lable and } \\
\text { negattve I s missing. }\end{array}$ \\
\hline 1201 & & $11 x 14$ & $1878 ?$ & $\begin{array}{l}\text { MAnerva TerTace, near VIaw, Meanoth Hot } \\
\text { Springs, Yollowstone Park. }\end{array}$ \\
\hline 2202 & & " & $1878 ?$ & $\begin{array}{l}\text { Columnar basalts capplag brink of Yellowetono } \\
\text { Canon, Tower Falls, Yellowstono Park. }\end{array}$ \\
\hline 1203 & & " & $1878 ?$ & $\begin{array}{l}\text { Orand Canon of the Yollowstone prom the foot } \\
\text { of the lower fall ahowlig the Red Planacle. } \\
\text { Yollow tone Paxix. }\end{array}$ \\
\hline 2204 & & B & $1878 ?$ & $\begin{array}{l}\text { Grand Canon of the Yellowstone, fron the brink } \\
\text { of the lower Ialls Yellowstone Park. }\end{array}$ \\
\hline 1205 & 179 & $n$ & $1872 ?$ & \\
\hline 1206 & 189 & $"$ & $1872 ?$ & \\
\hline 1207 & & $n$ & $1878 ?$ & $\begin{array}{l}\text { Great Falls of the Yollowetone, noar VIow. } \\
\text { Yellowstone Park. }\end{array}$ \\
\hline 1208 & & & & $\begin{array}{l}\text { Subject unknown. No print avaliable and } \\
\text { negative is missing. }\end{array}$ \\
\hline 1209 & & $" 1$ & $1872 ?$ & $\begin{array}{l}\text { Upper Farls of the Yellowetone and Upper Canon } \\
\text { Irow near brink of Great Falls, Yollowstone Park. }\end{array}$ \\
\hline
\end{tabular}




\begin{tabular}{|c|c|c|c|c|}
\hline $\begin{array}{l}\text { How } \\
\text { Nombass }\end{array}$ & $\begin{array}{l}\text { O2d } \\
\text { Catasos } \\
\text { Homber }\end{array}$ & 5280 & Dato & Reasriza \\
\hline 1210 & & & & $\begin{array}{l}\text { Subjoct unknowa. To print avaliable and } \\
\text { negative 10 alistng. }\end{array}$ \\
\hline 1212 & & & & 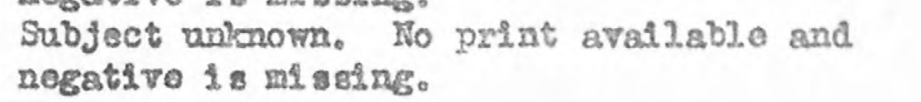 \\
\hline 1212 & 31 & $11 \pi 24$ & 1872 & \\
\hline 1213 & 29 & H & $"$ & \\
\hline 2224 & 21 & 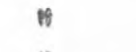 & 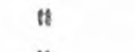 & \\
\hline 1225 & 9 & $n$ & " & \\
\hline 1216 & & $n$ & $1878 ?$ & $\begin{array}{l}\text { The Grand Tetons from noar hoad of Buffalo } \\
\text { Fork. Fyoming. }\end{array}$ \\
\hline 1227 & 8 & " & 1872 & \\
\hline 2218 & $6 \%$ & $n$ & $2872 ?$ & \\
\hline 122.9 & $5 ?$ & ต & $1872 ?$ & \\
\hline 1220 & 23 & " & 1872 & \\
\hline 1221 & & " & $1878 ?$ & $\begin{array}{l}\text { Mounts Doane and Langlord near Tollowstone } \\
\text { Lake, Yellowatone Park. }\end{array}$ \\
\hline 1222 & 72 & $"$ & $"$ & \\
\hline 1223 & 78 & " & " & \\
\hline 1224 & 74 & n & $n$ & \\
\hline $1224 \mathrm{a}$ & & $8 \pi 20$ & n & $\begin{array}{l}\text { The } 8 \times 10 \text { print } 1 \text { a from a reduction copg of } \\
\text { the orlglnal } 1122.4 \text { nogative, old maber } 7 A_{0} \\
2873 \text { gerles. }\end{array}$ \\
\hline 1225 & 35 & $11 \pi 14$ & 1872 & \\
\hline 2.225 & 38 & " & $n$ & \\
\hline $122 ?$ & 42 & $n$ & " & \\
\hline 1228 & 48 & H & 1873 & \\
\hline 1229 & 50 & $"$ & " & \\
\hline 1230 & 51 & $"$ & 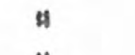 & \\
\hline 1231 & 65 & $"$ & " & \\
\hline 1232 & 56 & " & $n$ & \\
\hline 1233 & 60 & H & $"$ & \\
\hline 1234 & 65 & $n$ & $"$ & \\
\hline 1235 & 69 & $"$ & n & \\
\hline 1236 & 70 & N & $"$ & \\
\hline 1237 & 80 & $H$ & H & \\
\hline 1238 & 82. & " & $n$ & \\
\hline 1239 & 82 & n & $n$ & \\
\hline 1240 & 83 & $"$ & $"$ & \\
\hline 1241 & 85 & n & $n$ & . \\
\hline 1242 & 86 & " & * & $\cdot$ \\
\hline $12 A 3$ & 93 & $n$ & $n$ & \\
\hline $12 A 4$ & 99 & $n$ & ๓ & \\
\hline 1245 & 100 & " & $"$ & \\
\hline 1.246 & 202 & $n$ & $"$ & \\
\hline 1247 & 103 & $"$ & $m$ & \\
\hline 1248 & 106 & " & " & $\begin{array}{l}\text { This print } 1 \text { s Irom a copy nogative of an } \\
\text { enlarged portion of original } 11 \times 24 \text { negative. }\end{array}$ \\
\hline 1249 & $10 ?$ & " & $H$ & \\
\hline 1250 & 1 & " & 1872 & \\
\hline
\end{tabular}




\begin{tabular}{|c|c|c|c|c|}
\hline $\begin{array}{l}\text { New } \\
\text { Numbs? }\end{array}$ & $\begin{array}{l}\text { Old } \\
\text { Catalog } \\
\text { Thumber }\end{array}$ & $\mathrm{S1} 20$ & Date & Retrarks \\
\hline $\begin{array}{l}1251 \\
1252\end{array}$ & & $212 \times 24$ & $\underset{n}{2878}$ & 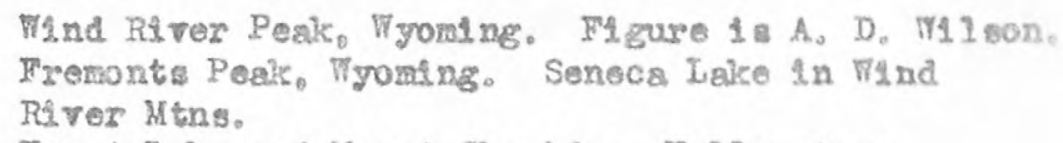 \\
\hline 1253 & & 11 & $"$ & $\begin{array}{l}\text { Heart Lake and Hount Sheridan, Yollowstoat } \\
\text { Nat1onal Park. }\end{array}$ \\
\hline 1254 & & " & & No caption for the ploture. \\
\hline $\begin{array}{l}1255 \\
2256\end{array}$ & & 1258 14 & & 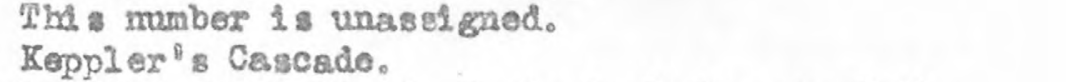 \\
\hline 1257 & & " & 1872 & 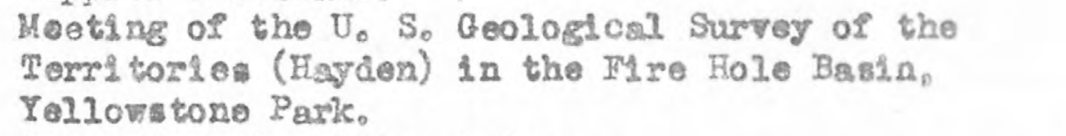 \\
\hline 1258 & & $8 \times 20$ & & Ho coptlor for thl pietura. \\
\hline 1259 & & $H$ & 18759 & $\begin{array}{l}\text { Laice San Cristoval, Finadale Coo Colorado. } \\
\text { This plcture may be } 8 \times 20 \text { reduction of old catsiog } \\
\text { no. I in 20x2A alzo of } 1875 \text { sertos. }\end{array}$ \\
\hline $1260^{\circ}$ & & " & $2875 ?$ & 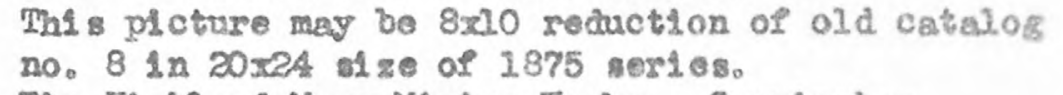 \\
\hline 2261 & & $"$ & & $\begin{array}{l}\text { The Highiand Mary M1alne Works. Cunntnghar } \\
\text { Gulch, Bakers Park. Colorado. }\end{array}$ \\
\hline 1262 & & $n$ & $2875 ?$ & $\begin{array}{l}\text { That ploture may be } 8 \times 10 \text { reduction of old eatalog } \\
\text { no. } 2 \text { in } 20 \times 24 \text { se of } 1875 \text { sertes. }\end{array}$ \\
\hline 1263 & & " & $1875 ?$ & $\begin{array}{l}\text { This plctraxe may be } 8 \text { selo reduction of old catelog } \\
\text { no. } 4 \text { in } 20 \times 24 \text { me of } 1875 \text { serses. }\end{array}$ \\
\hline 3264 & & " & $1875 ?$ & 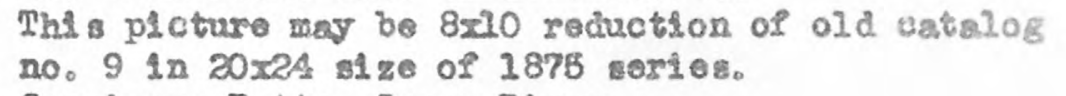 \\
\hline 1265 & & $n$ & & Gunnl wons Butte. Groen RIver. \\
\hline 2266 & & & & grai numbor is unasal gued。 \\
\hline 1267 & & $8 \geq 10$ & & The Book Clifls, near Green RIver, Utaho \\
\hline $\begin{array}{l}1268 \\
1269\end{array}$ & 273 & & 1871 & No caption for thl s plcture. \\
\hline 1270 & & 11 & & No caption for thls plcture. \\
\hline Unmambo: & red & $9 x 11 \frac{1}{2}$ & & Copy of Hount of Holy Cross. \\
\hline
\end{tabular}


, .
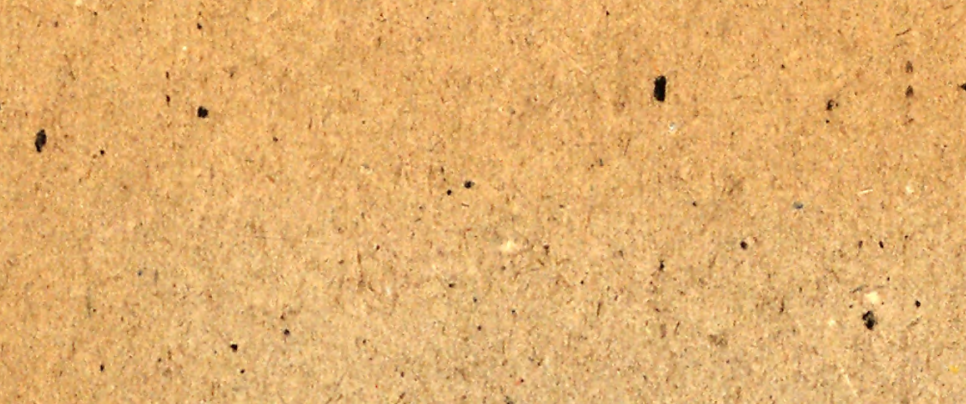

(4)

$46^{\circ}$

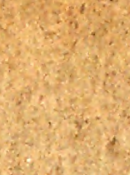

